\title{
TRIPLET EXTENSIONS I: \\ SEMIBOUNDED OPERATORS IN THE SCALE OF HILBERT SPACES
}

\author{
By \\ PAVEL KURASOV
}

\begin{abstract}
The extension theory for semibounded symmetric operators is generalized by including operators acting in a triplet of Hilbert spaces. We concentrate our attention on the case where the minimal operator is essentially self-adjoint in the basic Hilbert space and construct a family of its self-adjoint extensions inside the triplet. All such extensions can be described by certain boundary conditions, and a natural counterpart of Krein's resolvent formula is obtained.
\end{abstract}

\section{Introduction}

The extension theory for symmetric operators $[1,30]$ developed originally by J. von Neumann [27] gives an affirmative answer to the question under which conditions does a symmetric densely defined operator possess self-adjoint (canonical) extensions and describes all such extensions as restrictions of the adjoint operator. The family of self-adjoint operators may then be parametrized by Krein's resolvent formula [20], where spectral properties of the operators are encoded in a certain Nevanlinna function, usually known as Krein's $Q$-function.

In the current article, we study so-called triplet extensions of symmetric operators. Consider a triplet of Hilbert spaces [8] (see the rigorous definition below)

$$
G \subset H \subset G^{\dagger}
$$

and an operator $B$, which is symmetric and densely defined both as an operator in $G$ and in $H$. Certainly the deficiency indices for these two operators can be different; the case which attracted our attention is when the operator $B$ is semibounded and essentially self-adjoint in $H$, but has nontrivial deficiency indices as an operator in $G$. In this case, there is a unique self-adjoint extension of $B$ in $H$, but inside $G$ there is a nontrivial family of extensions. This family can be characterized by classical extension theory and therefore is not particular interesting. Such extensions do not 
fully use the structure of the Gelfand triplet (1.1); more precisely, the space $G^{\dagger}$ plays no role in this construction. On the other hand, every densely defined operator $B$ in $G$ determines the triplet adjoint operator $B^{\dagger}$ acting in $G^{\dagger}$. It is therefore interesting to study (generalized) extensions of $B$ which are at the same time restrictions of $B^{\dagger}$. We call such operators triplet extensions. This construction naturally generalizes the von Neumann approach and clearly coincides with it in the degenerate case $G=H=G^{\dagger}$. In defining triplet extensions, we exclude canonical extensions of $B$ in $G$, which can as well be obtained as restrictions of $B^{\dagger}$ since $B^{*} \subset B^{\dagger}$, where $B^{*}$ denotes the operator which is adjoint to $B$ in $G$ (see Definition 3.1 for details).

Starting with the most general definition of triplet extensions, we continue with the case where $G$ is one of the spaces from the scale of Hilbert spaces associated with the unique self-adjoint extension of $B$ in $H$. This assumption is satisfied in several examples exhibited below. In addition, we restrict our considerations to the case where the deficiency indices of $B$ in $G$ are $(1,1)$ in order to make our presentation more transparent; most of the formulas can easily be generalized to the case of any equal deficiency indices. For the same reason, we treat just the case when $B$ is essentially self-adjoint in $H$, since in this case triplet extensions are of particular interest. As expected, formulas generalizing Krein's resolvent formula play the central role in the characterization of the corresponding operator families. In particular, we obtain an extension of Krein's formula, where the role of the $Q$-function is played by a certain generalized Nevanlinna function. This is especially surprising, since the corresponding operator is self-adjoint in a certain Hilbert space, not in a Pontryagin space, where the appearance of generalized Nevanlinna functions is standard.

In the rest of the introduction, we discuss a few examples showing that triplet extensions are important in certain applications. The extension theory for symmetric operators plays an important role in modern mathematical physics, especially in quantum mechanics. This role is two-fold

- On the one hand, extension theory is sometimes needed to describe the family of self-adjoint operators corresponding to a formal differential expression obtained from a classical Hamiltonian via the correspondence principle.

- On the other hand, extension theory can be used to introduce interactions which are specific for quantum mechanics and do not have classical analogues, so-called contact interactions.

Probably the most important example connected with these two approaches concerns the Sturm-Liouville operator on the half-line, where in order to determine self-adjoint operators, one usually needs in addition to a formally symmetric differential expression certain boundary conditions at the origin. Then the spectral 
properties of the corresponding operators are described by the Titchmarsh-Weyl coefficient, which is identical to Krein's $Q$-function in this case - a certain Nevanlinna type function. It appears natural to try to generalize this approach in order to include the singular case (limit point case in accordance with H. Weyl's classification [30]) when the minimal operator has trivial deficiency indices. Almost all constructions considered so far lead to operators in Pontryagin spaces (with indefinite metrics) and generalized Nevanlinna functions [12, 14, 15, 24].

Another class of problems where we meet similar difficulties is the theory of singular interactions $[31,17,19,3,4,5,22,23,25,26]$, where one of the first questions is the rigorous definition of the operator formally given by

$$
L+\alpha\langle\varphi, \cdot\rangle \varphi, \quad \alpha \in \mathbb{R}
$$

where $L$ is a self-adjoint operator in the Hilbert space $H$ (with scalar product denoted by $\langle\cdot, \cdot\rangle)$ and $\varphi$ is a certain vector from the scale of Hilbert spaces $\mathcal{H}_{n}(L)$ associated with the self-adjoint operator $L$ (see the definition below). The interesting case occurs when $\varphi \notin \mathcal{H}$; otherwise, the perturbation term is just a bounded operator. One can define such a perturbation in the case $\varphi \in \mathcal{H}_{-n}, n=1,2$ by associating (1.2) with one of the self-adjoint extensions of the operator $L_{\min }$ the restriction of $L$ to the set of functions $\psi$ satisfying the additional condition $\langle\varphi, \psi\rangle=0$. The corresponding family of operators is again described by Krein's resolvent formula $[20,5]$ with a Nevanlinna function encoding their spectral properties. But this approach does not work in the case $\varphi \in \mathcal{H}_{-n} \backslash \mathcal{H}_{-2}, \quad n \geq 3$, because the corresponding restricted operator is essentially self-adjoint (in $H$ ), so that the original operator $L$ is its unique self-adjoint extension. Attempts to define a nontrivial family of operators in this case are again connected with generalized Nevanlinna functions and operators in Pontryagin spaces [14, 22].

It has been noted $[25,26,22]$ that the restricted operator has nontrivial deficiency indices considered in the Hilbert space $\mathcal{H}_{n-2}$ instead of $\mathcal{H}$. It follows that we may try to define the operator given formally by (1.2) as a triplet extension with respect to the triplet

$$
\mathcal{H}_{n-2} \subset H \subset \mathcal{H}_{-n+2},
$$

of Hilbert spaces from the scale associated with $L$ in $H$. The operator corresponding to (1.2) has been defined on a certain vector space of singular elements forming a sort of cascade belonging to different spaces from the scale [22, 12]. These elements belong to different spaces from the scale and therefore have different orders of singularity with respect to the operator $B$. It was shown that the vector space can be turned into a Hilbert space to obtain a family of self-adjoint operators 
corresponding to formal singular interactions. Unfortunately, this model is not optimal, in the sense that the parameters (certain normalization points $\mu_{j}<0$ and a Gram matrix $\Gamma$ ) have to be chosen satisfying certain restrictions whose origin was hard to understand. In particular, in order to obtain a Hilbert space model, it is necessary to choose all $\mu_{j}$ pairwise different, and $\Gamma$ cannot be chosen diagonal. These technical difficulties became an obstacle for further development of the theory and using it in applications. In the model presented here, the Gram matrix $\Gamma$ is diagonal, and an explicit explanation for the choice of normalization points $\mu_{j}$ is given. Since the basis elements in the new model have the same order of singularity, one may call it the peak model (in order to distinguish it from the cascade model given in [12]). This new model is more transparent and therefore more easily used in different applications. It has also been realized that the methods originally developed to interpret the formal expression (1.2) have much more general applications and can be used to construct triplet extensions. Such a general model is developed in the current article. It opens a new research field, giving a hint how to study the triplet extensions in the case of arbitrary deficiency indices. This program will be developed in a forthcoming article.

A similar approach has already been carried out for singular Sturm-Liouville operators of hydrogen atom type in [24] interpreting the generalized TitchmarshWeyl coefficient as Krein's $Q$-function, even in the case that it is of generalized Nevanlinna type.

The current article is organized as follows. As mentioned, our approach is a direct extension of the classical von Neumann theory or, more precisely, its version developed by M. Krein, M. Birman and M. Vishik [9, 21, 32, 6], which is very useful in the physical case of semibounded operators. This theory is briefly discussed in Section 2. The following two sections are devoted to the definition of triplet extensions in the general case and in the case where the triplet is formed just by three spaces from the scale. The minimal extended space is introduced. The corresponding minimal and maximal operators acting in the extended space are described in Section 5. The self-adjoint family of triplet extensions is finally obtained in Section 6 by restricting the maximal operator. The corresponding resolvents are calculated explicitly. In Section 7, we obtain a new extended resolvent formula and describe the class of functions appearing in its denominator. It is shown that these functions can also be obtained by a certain natural renormalization procedure demonstrated in Section 8. The last two sections are devoted to an application of the developed approach to the theory of singular perturbations. In particular, a new family of point interactions for the Laplacian in $\mathbb{R}^{3}$ is presented. 
In this article, we use the scale of Hilbert spaces

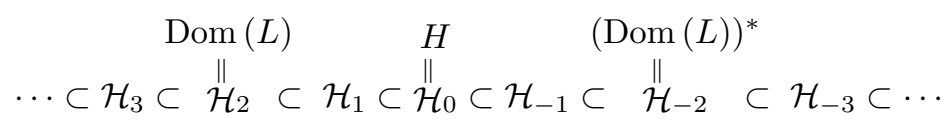

associated with a certain positive self-adjoint operator $L$. The spaces $\mathcal{H}_{-n}$, $n=1,2, \ldots$ can be considered as completions of $H=\mathcal{H}_{0}$ with respect to the norms

$$
\|U\|_{\mathcal{H}_{n}}^{2}=\left\langle U,(L+1)^{n} U\right\rangle
$$

where $\langle\cdot, \cdot\rangle=\langle\cdot, \cdot\rangle_{H}$ is the scalar product in the original Hilbert space $H$. Then the spaces with positive indices are just dual spaces

$$
\mathcal{H}_{s}=\mathcal{H}_{-s}^{*},
$$

so that the spaces $\mathcal{H}_{m} \subset H \subset \mathcal{H}_{-m}, m=1,2, \ldots$ form a Gelfand triplet (of Hilbert spaces). The operator $L+1$ acts as an isometric shift in the scale of Hilbert spaces mapping $\mathcal{H}_{n+2}$ onto $\mathcal{H}_{n}$. Let us denote by $L_{n}$ the restriction for $n>0$ and extension for $n<0$ of the operator $L$ to the domain $\operatorname{Dom}\left(L_{n}\right)=\mathcal{H}_{n+2}$. The operator $L_{n}$ so defined is self-adjoint in $\mathcal{H}_{n}$. In particular, the operator $L_{0}$ is equal to the operator $L$, and its domain is the space $\mathcal{H}_{2}$.

Note that an equivalent norm in $\mathcal{H}_{n}$ can be introduced using the scalar product

$$
\langle U, V\rangle_{\mathcal{H}_{n}}=\langle U, b(L) V\rangle_{H},
$$

where $b$ is any polynomial of order $n$ positive on $\mathbb{R}_{+} \cup\{0\}$. In what follows, we choose

$$
b(\lambda)=\left(\lambda-\mu_{1}\right)\left(\lambda-\mu_{2}\right) \cdots\left(\lambda-\mu_{m}\right),
$$

where $\mu_{1}, \ldots, \mu_{m}<0$ are arbitrary pairwise different negative numbers.

\section{Perturbations and extensions of semibounded opera- tors: classical theory}

The aim of this article is to generalize the extension theory for symmetric operators by including the case where the role of the adjoint operator is played by the triplet adjoint operator. It is clear that the classical extension theory, originally developed by J. von Neumann [27] (see also [10]), should appear as a special case when the triplet of Hilbert spaces degenerates into just one Hilbert space, so that we have $G=H=G^{\dagger}$. Since all operators appearing in this article are semibounded, it is natural to use a special version of the extension procedure given by M. Sh. Birman, 
M. G. Krein and M. I. Vishik [9, 21, 32] and usually called Birman-Krein-Vishik theory [6]. In this section, we recall the main ideas of this approach.

Let $B$ be a nonnegative closed symmetric densely defined operator acting in a Hilbert space $H$. Assume that it has deficiency indices $(1,1)$. Then the Hilbert space possesses the decomposition

$$
H=\operatorname{Range}(B-\mu) \oplus \operatorname{Ker}\left(B^{*}-\mu\right), \quad \mu \in \mathbb{R}_{-},
$$

where $B^{*}$ denotes the operator adjoint to $B$ in the space $H$ and the negative parameter $\mu$ can be chosen arbitrarily. Then any self-adjoint extension $A$ is at the same time a restriction of the adjoint operator, so that the formula

$$
B \subset A \subset B^{*}
$$

holds. We denote by $A$ any nonnegative self-adjoint extension of $B$. Such an extension can be obtained, for example, by closing the domain of $B$ with respect to the graph norm. This extension is usually called the Friedrichs extension, but the role of $A$ can be played by any other nonnegative extension.

Let us denote by $G(\lambda)$ the family of deficiency elements satisfying the identity

$$
G(\lambda)=\frac{A-\mu}{A-\lambda} G(\mu)=G(\mu)+(\lambda-\mu) \frac{1}{A-\lambda} G(\mu), \quad \lambda, \mu \in \mathbb{C} \backslash \mathbb{R}_{+} .
$$

Then the domain of the adjoint operator is given by

$$
\operatorname{Dom}\left(B^{*}\right)=\operatorname{Dom}(A) \dot{+} \mathcal{L}\{G(\mu)\},
$$

where $\mu<0$ is a certain fixed point on the negative half-axis and $\mathcal{L}$ denotes the linear span. The sum here is direct, since $G(\mu) \notin \operatorname{Dom}(A)$ and therefore every $U \in \operatorname{Dom}\left(B^{*}\right)$ can be written as

$$
U=U_{r}+u G(\mu), \quad U_{r} \in \operatorname{Dom}(A), \quad u \in \mathbb{C},
$$

and this representation is unique. The action of the operator $B^{*}$ is given by

$$
B^{*}\left(U_{r}+u G(\mu)\right)=A U_{r}+\mu u G(\mu) .
$$

The domain of the maximal operator can also be described as

$$
\operatorname{Dom}\left(B^{*}\right)=\operatorname{Dom}(A)+\mathcal{L}\left\{G(\lambda): \lambda \in \mathbb{C} \backslash \mathbb{R}_{+}\right\},
$$

where the parameter $\lambda$ runs over all complex numbers excluding the positive halfaxis. Note that in this representation, the sum is no longer direct, since

$$
G(\mu)-G(\lambda) \in \operatorname{Dom}(A) .
$$


This formula shows that (2.4) and (2.7) describe exactly the same linear sets.

Now every self-adjoint restriction of the maximal operator can be described by imposing the boundary condition

$$
\left\langle G(\mu),(A-\mu) U_{r}\right\rangle=\gamma u, \quad \gamma \in \mathbb{R} \cup\{\infty\}
$$

on the functions possessing representation (2.5). Let us denote the corresponding operator by $A_{\gamma}$. In this parametrization, we have $A=A_{\infty}$. The boundary condition guarantees that the resolvent equation

$$
\left(A_{\gamma}-\lambda\right) U=F, \quad F \in H
$$

is solvable for all $\lambda \in \mathbb{C} \backslash \mathbb{R}$, and this solution leads to Krein's resolvent formula

$$
\frac{1}{A_{\gamma}-\lambda}=\frac{1}{A-\lambda}-\frac{1}{\langle G(\mu),(\lambda-\mu) G(\lambda)\rangle-\gamma}\langle G(\bar{\lambda}), \cdot\rangle G(\lambda) .
$$

In this formula, the function

$$
Q(\lambda)=\langle G(\mu),(\lambda-\mu) G(\lambda)\rangle
$$

is a Nevanlinna function, i.e., is analytic outside the real axis, symmetric with respect to the real axis and has nonnegative imaginary part in the upper half-plane. This function is usually called Krein's $Q$-function, and the parameter $\gamma$ describes all possible self-adjoint extensions of $B$. Note that, in general, the parameter $\gamma$ depends on the chosen regularization point $\mu$.

\section{Self-adjoint extensions in a triplet of Hilbert spaces}

Consider a triplet of Hilbert spaces

$$
G \subset H \subset G^{\dagger}
$$

satisfying the following properties:

- $G, H, G^{\dagger}$ are Hilbert spaces;

- the space $G$ is a dense subspace of $H$;

- the space $H$ is a dense subspace of $G^{\dagger}$;

- the space $G^{\dagger}$ is dual to $G$ with respect to the norm in $H$.

It is natural to extend the notation $\langle\cdot, \cdot\rangle_{H}$, denoting originally the scalar product in $H$, to the pairing between elements from $G$ and $G^{\dagger}$, so that

$$
\langle U, V\rangle_{H}
$$


is well-defined whenever $U \in G, V \in G^{\dagger}$ or $U \in G^{\dagger}, V \in G$. Let $B$ be a densely defined operator in $G$. Then the triplet adjoint operator $B^{\dagger}$ is defined in the Hilbert space $G^{\dagger}$ on the domain

$$
\operatorname{Dom}\left(B^{\dagger}\right)=\left\{V \in G^{\dagger}: \exists C_{V}>0: U \in \operatorname{Dom}(B) \Rightarrow\left|\langle V, B U\rangle_{H}\right| \leq C_{V}\|U\|_{G}\right\},
$$

by the equality

$$
\langle V, B U\rangle_{H}=\left\langle B^{\dagger} V, U\right\rangle_{H}, \quad \text { for all } U \in \operatorname{Dom}(B) .
$$

Here we investigate so-called triplet extensions of symmetric operators $B$ in $G$ having nontrivial deficiency indices. We consider the case where $B$ is semibounded and has defect one. Our main interest lies in the situation in which the operator $B$ is essentially self-adjoint in $H$, i.e., its closure in $H$ is a self-adjoint operator. In this case, considering $B$ in $H$ does not lead to an interesting extension theory, and triplet extensions start to play an important role.

By a triplet extension of $B$ we understand a generalized extension of $B$ to a certain Hilbert space $\mathbf{H}$ inside $G^{\dagger}$ which is simultaneously a restriction of the triplet adjoint operator $B^{\dagger}$. In order to exclude canonical extensions (inside $G$ ), we assume that $\mathbf{H}$ contains $\operatorname{Ker}\left(B^{\dagger}-\mu\right)$, for all $\mu \in \mathbb{C} \backslash R_{+}$. More precisely, we have the following definition

Definition 3.1. Let $G \subset H \subset G^{\dagger}$ be a triplet of Hilbert spaces and let $B$ be a densely defined symmetric operator in $G$. An operator A acting in a Hilbert space $\mathbf{H}$ is a self-adjoint triplet extension of the operator $B$ if and only if

- the space $\mathbf{H}$

(1) is a subset of $G^{\dagger}$;

(2) contains $G$ as a Hilbert subspace;

(3) contains $\operatorname{Ker}\left(B^{\dagger}-\mu\right)$, for all $\mu \in \mathbb{C} \backslash \mathbb{R}_{+}$;

i.e.,

$$
G \subset \mathbf{H} \subset G^{\dagger},
$$

and

- the operator $\mathbf{A}$ is self-adjoint in $\mathbf{H}$ and satisfies

$$
B \subset \mathbf{A} \subset B^{\dagger},
$$

i.e., it is an extension of $B$ and a restriction of $B^{\dagger}$.

Formula (3.2) follows directly from assumptions (1) and (2) and implies together with (3.3) that every triplet extension of $B$ is an operator acting inside the 
triplet and this operator acts as the triplet adjoint. This is a direct generalization of the classical formula (2.2) valid for self-adjoint extensions inside the space (when the triplet (3.1) reduces to just one Hilbert space).

If the operator $B$ in $G$ has nontrivial deficiency indices, then the kernel $\operatorname{Ker}\left(B^{\dagger}-\lambda\right)$ is always nontrivial. In particular, Definition 3.1 implies that

$$
\mathbf{H} \supset \text { Range }(B-\mu) \dot{+} \operatorname{Ker}\left(B^{\dagger}-\mu\right), \quad \mu \in \mathbb{R}_{-},
$$

where the sum is orthogonal with respect to the scalar product in $G^{\dagger}$ (but not necessarily in $\mathbf{H}$ ). This formula is a natural generalization of (2.1) valid for extensions inside the space.

If $B$ is essentially self-adjoint in $G$, then the kernel $\operatorname{Ker}\left(B^{\dagger}-\mu\right), \mu \in \mathbb{R}_{-}$, is trivial, since $B^{\dagger}$ is a positive self-adjoint operator in $G^{\dagger}$. Condition (3) makes no further restriction on $\mathbf{H}$ in this case, and the closure of $B$ in $H$ satisfies the definition. Therefore in what follows only operators with nontrivial deficiency indices will be considered.

We do not aim to describe the whole family of triplet extensions in the current article. The family we construct is minimal in the sense that the space $\mathbf{H}$ is the minimal vector space satisfying assumptions (1)-(3). In addition, we assume that the spaces $G$ and $G^{\dagger}$ are from the scale of Hilbert spaces associated with a certain nonnegative operator and $B$ is a restriction of this operator to a subspace of $G$. The corresponding triplet extensions can be referred to as extensions in the scale of Hilbert spaces and are considered further in the following section.

\section{Triplet extensions in the scale of Hilbert spaces}

In this section, we consider the case in which the Hilbert space $G$ from the triplet (3.1) coincides with one of the Hilbert spaces associated with the closure of $B$ in $H$ (recall that $B$ is essentially self-adjoint in $H$ ). It is convenient to change our point of view slightly. Let $L$ be a nonnegative self-adjoint operator in the Hilbert space $H$. Consider the triplet

$$
\mathcal{H}_{m} \subset H \subset \mathcal{H}_{-m}
$$

and the minimal operator $L_{\min }$ satisfying the conditions

(1) $L_{\min }$ is a restriction of $L$;

(2) $L_{\text {min }}$ has deficiency indices $(1,1)$ in $\mathcal{H}_{m}$;

(3) $L_{\min }$ is essentially self-adjoint in $H$.

It follows that $L_{\text {min }}$ is a restriction of $L_{m}$, which is self-adjoint in $\mathcal{H}_{m}$. The triplet adjoint to the operator $L_{m}$ coincides with $L_{-m}$. It follows that the maximal operator $L_{\text {max }}=L_{\text {min }}^{\dagger}$, the triplet adjoint to $L_{\text {min }}$, is an extension of $L_{-m}$ in $\mathcal{H}_{-m}$. 
Hence we are in the situation described at the beginning of the preceding section with

$$
G=\mathcal{H}_{m}, \quad G^{\dagger}=\mathcal{H}_{-m}, \quad B=L_{\min } \quad \text { and } \quad B^{\dagger}=L_{\text {max }} .
$$

In what follows, we investigate the possibility of constructing a self-adjoint triplet extension $\mathbb{A}$ of $L_{\min }$ in a certain Hilbert space $\mathbb{H}$. In our approach, such extensions are constructed by first specifying the linear space $\mathbb{H}$ and then defining the operator $\mathbb{A}_{\max }$ in it as a restriction of $L_{\max }$. Such an operator possesses self-adjoint restrictions if and only if the minimal operator $\mathbb{A}_{\min }=\mathbb{A}_{\max }^{*}$ - the adjoint of $\mathbb{A}_{\max }$ in $\mathbb{H}$ - is also a restriction of $\mathbb{A}_{\max }$. We show that $\mathbb{A}_{\min }$ is also an extension of $L_{\min }$, so that the following inclusions hold:

$$
L_{\min } \subset \mathbb{A}_{\min } \subset \mathbb{A}_{\max } \subset L_{\max }
$$

This condition implies certain restrictions on the scalar product, which now may be introduced on $\mathbb{H}$ to turn it into a Hilbert space. If formula (4.2) holds, then a triplet extension $\mathbb{A}$ of $L_{\text {min }}$ can be obtained using standard extension theory inside the new Hilbert space $\mathbb{H}$ so that

$$
L_{\min } \subset \mathbb{A}_{\min } \subset \mathbb{A} \subset \mathbb{A}_{\max } \subset L_{\max }
$$

Let us denote by $G(\lambda)$ the family of deficiency elements for the operator $L_{\min }$ in $\mathcal{H}_{m}$ which are solutions to the equation $\left(L_{\text {min }}^{*}-\lambda\right) G(\lambda)=0 .{ }^{1}$ Then the self-adjoint canonical extensions of $L_{\text {min }}$ inside the space $\mathcal{H}_{m}$ can be constructed following the original scheme described in Section 2; in particular, formula (2.4) implies

$$
\operatorname{Dom}\left(L_{\text {min }}^{*}\right)=\operatorname{Dom}\left(L_{m}\right) \dot{+} \mathcal{L}\{G(\mu)\}, \quad \mu<0 .
$$

This construction does not bring any new ideas, and the corresponding operator is not a triplet extension of $L_{\text {min }}$ since condition 3 in Definition 3.1 is not satisfied.

Let us determine the domain of the maximal operator $L_{\max }=L_{\min }^{\dagger}$. The domain of $L_{\text {min }}^{\dagger}$ consists of all $V \in \mathcal{H}_{-m}$ such that the form $\langle V, L U\rangle_{H}, U \in \operatorname{Dom}\left(L_{\text {min }}\right)$, determines a bounded linear functional with respect to $U \in \mathcal{H}_{m}$, i.e.,

$$
\left|\langle V, L U\rangle_{H}\right| \leq C_{V}\|U\|_{\mathcal{H}_{m}}, \quad \text { for some } C_{V}>0
$$

Recall that the domain of $L_{\text {min }}^{*}$ described by (4.4) is precisely the set of all $W \in \mathcal{H}_{m}$ such that

$$
\left|\langle W, L U\rangle_{\mathcal{H}_{m}}\right|=\left|\langle W, b(L) L U\rangle_{H}\right| \leq \tilde{C}_{W}\|U\|_{\mathcal{H}_{m}}, \quad \text { for some } \tilde{C}_{W}>0 .
$$

\footnotetext{
${ }^{1} L_{\text {min }}^{*}$ denotes here the operator adjoint to $L_{\text {min }}$ in the Hilbert space $\mathcal{H}_{m}$.
} 
It follows that $\operatorname{Dom}\left(L_{\max }\right)=b(L) \operatorname{Dom}\left(L_{\text {min }}^{*}\right)$; and hence every $V \in \operatorname{Dom}\left(L_{\text {max }}\right)$ possesses the representation

$$
V=\tilde{V}+v g(\mu), \quad \tilde{V} \in \mathcal{H}_{-m+2}, \quad v \in \mathbb{C},
$$

where

$$
g(\mu)=b(L) G(\mu) \in \mathcal{H}_{-m}
$$

and $\mu$ is a fixed negative number. Since the operators $L$ and $b(L)$ commute, the action of $L_{\max }$ is given by

$$
L_{\max }(\tilde{V}+v g(\mu))=L \tilde{V}+\mu v g(\mu)
$$

In particular, the function $g(\lambda), \lambda \in \mathbb{C} \backslash \mathbb{R}_{+}$solves the equation

$$
\left(L_{\max }-\lambda\right) g(\lambda)=0
$$

and every other solution is a multiple of $g(\lambda)$. The functions $g(\lambda)$ resemble the deficiency elements appearing in the classical extension theory but do not belong to the original Hilbert space $\mathcal{H}$, since the operator $L_{\min }$ is essentially self-adjoint there. $^{2}$ In our approach, $g(\lambda)$ is a deficiency element for the minimal operator defined in a certain extension of the original Hilbert space.

Lemma 4.1. In the above situation, the minimal vector space $\mathbb{H}$ satisfying assumptions 1, 2 and 3 of Definition 3.1 is an m-dimensional extension of $\mathcal{H}_{m}$, which can be described as

$$
\mathbb{H}=\mathcal{H}_{m} \dot{+} \mathcal{L}\left\{g\left(\mu_{1}\right), g\left(\mu_{2}\right), \ldots, g\left(\mu_{m}\right)\right\},
$$

where $\mu_{j}$ are different negative numbers

$$
\mu_{j}<0, \quad \mu_{j} \neq \mu_{i}, \quad i, j=1,2, \ldots, m .
$$

Proof. The set $\mathbb{H}$ contains at least the set

$$
\mathcal{H}_{m}+\mathcal{L}\left\{g(\lambda), \lambda \in \mathbb{C} \backslash R_{+}\right\}
$$

We claim that this extension is finite dimensional. It is sufficient to show that every $g(\lambda)$ can be written as a linear combination of $g\left(\mu_{j}\right), j=1, \ldots, m$ and a function from $\mathcal{H}_{m}$. Indeed, this representation is given by

$$
g(\lambda)=\sum_{j=1}^{m} \frac{b_{j}(\lambda)}{b_{j}\left(\mu_{j}\right)} g\left(\mu_{j}\right)+b(\lambda) G(\lambda),
$$

\footnotetext{
${ }^{2}$ In fact, $g(\lambda)$ is a deficiency element for the operator $L_{-m}$ restricted to the set of functions $U \in \mathcal{H}_{-m+2}$ satisfying the further condition $\langle U,(L-\lambda) G(\lambda)\rangle=0$.
} 
where the polynomial $b$ is determined by (1.6) and

$$
b_{j}(\lambda)=\prod_{i \neq j}\left(\lambda-\mu_{i}\right) .
$$

The sum in (4.8) is direct, since

$$
\mathcal{H}_{m} \cap \mathcal{L}\left\{g\left(\mu_{1}\right), \ldots, g\left(\mu_{m}\right)\right\}=\{0\} .
$$

Hence every vector space satisfying the assumptions of Definition 3.1 contains the vector space given by (4.8).

The last lemma describes $\mathbb{H}$ as a vector space. It can be turned into a Hilbert space by introducing a scalar product using a certain positive definite Gram matrix $\Gamma$

$$
\langle\mathbb{U}, \mathbb{V}\rangle_{\mathbb{H}}=\langle U, b(L) V\rangle_{H}+\langle\vec{u}, \Gamma \vec{v}\rangle_{\mathbb{C}^{m}},
$$

where we write

$$
\vec{u}=\left(u_{1}, u_{2}, \ldots, u_{m}\right) \in \mathbb{C}^{m} .
$$

With this scalar product, the Hilbert space $\mathbb{H}$ can be identified with the orthogonal sum

$$
\mathbb{H} \cong \mathcal{H}_{m} \oplus \mathbb{C}^{m},
$$

with the natural identification

$$
\mathbb{U}=(U, \vec{u})=U+\sum_{j=1}^{m} u_{j} g\left(\mu_{j}\right) .
$$

Therefore, in what follows, elements from $\mathbb{H}$ are considered both as functions from $\mathcal{H}_{-m}$ and as pairs $\mathbb{U}=(U, \vec{u}), U \in \mathcal{H}_{m}, \vec{u} \in \mathbb{C}^{m}$.

It turns out that the matrix $\Gamma$ has to be chosen diagonal in order to satisfy (4.2); but, in order to explore all possibilities, we assume for the moment that $\Gamma$ is just a Hermitian matrix with positive eigenvalues.

\section{Maximal and minimal operators}

In this section, we describe the maximal and minimal operators acting in the extension space $\mathbb{H}$. The operator $\mathbb{A}_{\max }$ acting in $\mathbb{H}$ is defined as the restriction of the linear operator $L_{\max }$ to the space $\mathbb{H}$. 
Lemma 5.1. Let $\mathbb{A}_{\max }$ be the restriction of $L_{\max }$ to the space $\mathbb{H}$. Then it acts on the domain

$$
\begin{aligned}
\operatorname{Dom}\left(\mathbb{A}_{\text {max }}\right) & =\mathcal{H}_{m+2} \dot{+} \mathcal{L}\left\{g(\mu), g\left(\mu_{1}\right), g\left(\mu_{2}\right), \ldots, g\left(\mu_{m}\right)\right\} \ni \mathbb{U} \\
\mathbb{U} & =U_{r}+u g(\mu)+\sum_{j=1}^{m} u_{j} g\left(\mu_{j}\right), \quad U_{r} \in \mathcal{H}_{m+2}, u, u_{j} \in \mathbb{C},
\end{aligned}
$$

as

$$
\mathbb{A}_{\max }\left(U_{r}+u g(\mu)+\sum_{j=1}^{m} u_{j} g\left(\mu_{j}\right)\right)=L U_{r}+\mu u g(\mu)+\sum_{j=1}^{m} \mu_{j} u_{j} g\left(\mu_{j}\right),
$$

where $\mu \neq \mu_{j}$ is a certain negative number and $\mu_{j}$ satisfy (4.9).

Proof. By definition, the domain of $\mathbb{A}_{\max }$ is given by

$$
\operatorname{Dom}\left(\mathbb{A}_{\max }\right)=\left\{\mathbb{U} \in \mathbb{H} \cap \operatorname{Dom}\left(L_{\max }\right): L_{\max } \mathbb{U} \in \mathbb{H}\right\},
$$

where the first condition actually represents no restriction since every $\mathbb{U} \in \mathbb{H}$ possesses the representation (4.5)

$$
\mathbb{U}=U+\sum_{j=1}^{m} u_{j} g\left(\mu_{j}\right), \quad U \in \mathcal{H}_{m}, \quad u_{j} \in \mathbb{C}
$$

and therefore belongs to Dom $\left(L_{\max }\right)$. Hence such a function belongs to the domain of $\mathbb{A}_{\max }$ if and only if it is mapped by the operator $L_{\max }-\mu, \mu<0$, to a certain $\mathbb{V} \in \mathbb{H}$. Since $\mathbb{V}$ again possesses the representation (4.15), we have

$$
(L-\mu) U+\sum_{j=1}^{m} u_{j}\left(\mu_{j}-\mu\right) g\left(\mu_{j}\right)=V+\sum_{j=1}^{m} v_{j} g\left(\mu_{j}\right)
$$

which implies

$$
U=(L-\mu)^{-1} V+\sum_{j=1}^{m}\left(v_{j}-u_{j}\left(\mu_{j}-\mu\right)\right)(L-\mu)^{-1} g\left(\mu_{j}\right) .
$$

Every such function $U$ can be written as a linear combination of the functions

$$
g(\mu), g\left(\mu_{1}\right), g\left(\mu_{2}\right), \ldots, g\left(\mu_{m}\right)
$$

and a function from $\mathcal{H}_{m+2}$. It follows that $\mathbb{U}$ possesses the representation (5.1). It is clear that the sum is direct, since no nontrivial linear combination of $g(\mu)$, $g\left(\mu_{1}\right), \ldots, g\left(\mu_{m}\right)$ belongs to $\mathcal{H}_{m+2}$.

Taking into account that $L_{\max } g(\lambda)=\lambda g(\lambda)$ and $L_{\max } U_{r}=L U_{r}$, we get formula (5.2). 
We have shown that the maximal operator $\mathbb{A}_{\max }$ is given by formulas (5.1) and (5.2). In what follows it is useful to obtain a description of the domain and the action of this operator compatible with the orthogonal decomposition (4.14).

Let us introduce the diagonal $m \times m$ matrix

$$
\mathcal{M}=\operatorname{diag}\left\{\mu_{1}, \mu_{2}, \ldots, \mu_{m}\right\}
$$

and the $m$-dimensional vector $\vec{b} \in \mathbb{C}^{m}$ with coordinates

$$
b^{j}=\frac{1}{b_{j}\left(\mu_{j}\right)}, \quad j=1,2, \ldots, m,
$$

where the polynomials $b_{j}$ are given by (4.11).

Lemma 5.2. For the maximal operator $\mathbb{A}_{\max }$ given by (5.1) and (5.2), it holds that

(5.5) $\operatorname{Dom}\left(\mathbb{A}_{\max }\right)=\left\{\mathbb{U}=(U, \vec{u}): U=U_{r}+u G(\mu), U_{r} \in \mathcal{H}_{m+2}, u \in \mathbb{C}, \vec{u} \in \mathbb{C}^{m}\right\}$

and

$$
\mathbb{A}_{\max }\left(\begin{array}{c}
U_{r}+u G(\mu) \\
\vec{u}
\end{array}\right)=\left(\begin{array}{c}
L U_{r}+\mu u G(\mu) \\
\mathcal{M} \vec{u}+u \vec{b}
\end{array}\right) .
$$

Proof. It follows from

$$
G(\mu)=\sum_{j=1}^{m} \frac{1}{b_{j}\left(\mu_{j}\right)} \frac{1}{\mu_{j}-\mu}\left(g\left(\mu_{j}\right)-g(\mu)\right) \in \mathcal{H}_{m}
$$

that any function $\mathbb{U}$ from $\operatorname{Dom}\left(\mathbb{A}_{\max }\right)$ given by (5.1) can be written in the form

$$
U_{r}+u G(\mu)+\sum_{j=1}^{m} u_{j} g\left(\mu_{j}\right), \quad U_{r} \in \mathcal{H}_{m+2}
$$

with coefficients $u, u_{j} \in \mathbb{C}$. Hence formula (5.5) holds.

Let us calculate the action of the operator. Using (5.7) and (5.2), we get

$$
\begin{aligned}
\mathbb{A}_{\max }\left(U_{r}\right. & \left.+u G(\mu)+\sum_{j=1}^{m} u_{j} g\left(\mu_{j}\right)\right) \\
& =\mathbb{A}_{\max }\left(U_{r}+u \sum_{j=1}^{m} \frac{1}{b_{j}\left(\mu_{j}\right)} \frac{1}{\mu-\mu_{j}}\left(g(\mu)-g\left(\mu_{j}\right)\right)+\sum_{j=1}^{m} u_{j} g\left(\mu_{j}\right)\right) \\
& =L U_{r}+u \sum_{j=1}^{m} \frac{1}{b_{j}\left(\mu_{j}\right)} \frac{1}{\mu-\mu_{j}}\left(\mu g(\mu)-\mu_{j} g\left(\mu_{j}\right)\right)+\sum_{j=1}^{m} u_{j} \mu_{j} g\left(\mu_{j}\right) \\
& =L U_{r}+\mu u G(\mu)+\sum_{j=1}^{m}\left(\mu_{j} u_{j}+u \frac{1}{b_{j}\left(\mu_{j}\right)}\right) g\left(\mu_{j}\right)
\end{aligned}
$$

which completes the proof. 
The maximal operator $\mathbb{A}_{\max }$ plays the role of the adjoint operator for the extension problem in $\mathbb{H}$. In the sequel, we require its boundary form, which shows in particular that this operator is not symmetric:

$$
\begin{aligned}
& \left\langle\mathbb{A}_{\max } \mathbb{U}, \mathbb{V}\right\rangle_{\mathbb{H}}-\left\langle\mathbb{U}, \mathbb{A}_{\max } \mathbb{V}\right\rangle_{\mathbb{H}} \\
= & \left\langle\left(\begin{array}{c}
L U_{r}+\mu u G(\mu) \\
\mathcal{M} \vec{u}+u \vec{b}
\end{array}\right),\left(\begin{array}{c}
V_{r}+v G(\mu) \\
\vec{v}
\end{array}\right)\right\rangle_{\mathbb{H}}-\left\langle\left(\begin{array}{c}
U_{r}+u G(\mu) \\
\vec{u}
\end{array}\right),\left(\begin{array}{c}
L V_{r}+\mu v G(\mu) \\
\mathcal{M} \vec{v}+v \vec{b}
\end{array}\right)\right\rangle_{\mathbb{H}} \\
= & \left\langle L U_{r}+\mu u G(\mu), b(L)\left(V_{r}+v G(\mu)\right)\right\rangle_{H}-\left\langle U_{r}+u G(\mu), b(L)\left(L V_{r}+\mu v G(\mu)\right)\right\rangle_{H} \\
& +\langle\mathcal{M} \vec{u}+u \vec{b}, \Gamma \vec{v}\rangle_{\mathbb{C}^{m}}-\langle\vec{u}, \Gamma(\mathcal{M} \vec{v}+v \vec{b})\rangle_{\mathbb{C}^{m}} \\
= & \bar{u}\left(\langle\Gamma \vec{b}, \vec{v}\rangle_{\mathbb{C}^{m}}-\left\langle g(\mu),(L-\mu) V_{r}\right\rangle_{H}\right)-\frac{\left(\langle\Gamma \vec{b}, \vec{u}\rangle_{\mathbb{C}^{m}}-\left\langle g(\mu),(L-\mu) U_{r}\right\rangle_{H}\right) v}{} \\
& +\langle\vec{u},(\mathcal{M} \Gamma-\Gamma \mathcal{M}) \vec{v}\rangle_{\mathbb{C}^{m}} .
\end{aligned}
$$

Note that if $\Gamma$ is diagonal, the last term in the formula vanishes.

Any triplet extension of the operator $L_{\min }$ can now be characterized as a selfadjoint restriction of $\mathbb{A}_{\max }$. Consider the minimal operator $\mathbb{A}_{\min }$ acting in $\mathbb{H}$ — the operator adjoint to $\mathbb{A}_{\max }$ in $\mathbb{H}$. The operator $\mathbb{A}_{\max }$ possesses symmetric restrictions if and only if the minimal operator $\mathbb{A}_{\text {min }}$ is symmetric or, in other words, is a restriction of the maximal operator. This necessary property of the new minimal operator puts certain restrictions on the Gram matrix $\Gamma$ which defines the scalar product in $\mathbb{H}$. Knowing that the new minimal operator $\mathbb{A}_{\min }$ is symmetric enables us to determine all triplet extensions of $L_{\min }$ using standard extension theory as described in Section 2.

Let us now calculate the minimal operator $\mathbb{A}_{\text {min }}$ for arbitrary choice of the Gram matrix $\Gamma$.

Lemma 5.3. The operator $\mathbb{A}_{\min }$ is defined on the functions in $\operatorname{Dom}\left(\mathbb{A}_{\max }\right)$ (given by (5.5)), which satisfy the two additional conditions

$$
\left\{\begin{array}{l}
u=0, \\
\langle\Gamma \vec{b}, \vec{u}\rangle_{\mathbb{C}^{m}}=\left\langle g(\mu),(L-\mu) U_{r}\right\rangle_{H}
\end{array}\right.
$$

and acts as

$$
\mathbb{A}_{\min }\left(\begin{array}{c}
U_{r} \\
\vec{u}
\end{array}\right)=\left(\begin{array}{c}
L U_{r} \\
\Gamma^{-1} \mathcal{M} \Gamma \vec{u}
\end{array}\right) .
$$

Proof. We calculate the operator adjoint to $\mathbb{A}_{\max }-\mu$. Consider two arbitrary vectors, $\mathbb{U} \in \operatorname{Dom}\left(\mathbb{A}_{\max }\right)$ and $\mathbb{V} \in \mathbb{H}$. The sesquilinear form of the operator 
$\mathbb{A}_{\max }-\mu$ is

$$
\left\langle\mathbb{V},\left(\mathbb{A}_{\max }-\mu\right) \mathbb{U}\right\rangle_{\mathbb{H}}=\left\langle V, b(L)(L-\mu) U_{r}\right\rangle_{H}+\langle\vec{v}, \Gamma(\mathcal{M}-\mu) \vec{u}\rangle_{\mathbb{C}^{m}}+\overline{\langle\vec{b}, \Gamma \vec{v}\rangle_{\mathbb{C}^{m}} u} .
$$

Consider first vectors of the type $\mathbb{U}=\left(U_{r}, \overrightarrow{0}\right)$; then the sesquilinear form reduces to

$$
\left\langle\mathbb{V},\left(\mathbb{A}_{\max }-\mu\right) \mathbb{U}\right\rangle_{\mathbb{H}}=\left\langle V, b(L)(L-\mu) U_{r}\right\rangle_{H}
$$

and determines a bounded linear functional with respect to $\mathbb{U}$ in the norm of $\mathbb{H}$ only if $V=V_{r} \in \mathcal{H}_{m+2}$. Using this fact, we can now write the boundary form for arbitrary $\mathbb{U} \in \operatorname{Dom}\left(\mathbb{A}_{\max }\right)$ as

$$
\begin{aligned}
\left\langle\mathbb{V},\left(\mathbb{A}_{\max }-\mu\right) \mathbb{U}\right\rangle_{\mathbb{H}}= & \left\langle V, b(L)(L-\mu)\left(U_{r}+u G(\mu)\right)\right\rangle_{H}+\langle(\mathcal{M}-\mu) \Gamma \vec{v}, \vec{u}\rangle_{\mathbb{C}^{m}} \\
& +\overline{\left\{\langle\vec{b}, \Gamma \vec{v}\rangle_{\mathbb{C}^{m}}-\left\langle G(\mu),(L-\mu) b(L) V_{r}\right\rangle_{H}\right\}} u .
\end{aligned}
$$

The first two summands determine bounded linear functionals with respect to $\mathbb{U} \in \mathbb{H}$, but the functional $\left(U_{r}+u G(\mu), \vec{u}\right) \mapsto u$ is not bounded in the norm of $\mathbb{H}$. Thus, the expression in the curly brackets must vanish. In other words, every function $\mathbb{V} \in \operatorname{Dom}\left(\mathbb{A}_{\min }\right)$ should satisfy

$$
\langle\Gamma \vec{b}, \vec{v}\rangle_{\mathbb{C}^{m}}=\left\langle g(\mu),(L-\mu) V_{r}\right\rangle_{H} .
$$

Summing up, we have proved that the domain of the adjoint operator $\mathbb{A}_{\min }-\mu$ is determined by (5.9) and the sesquilinear form is

$$
\left\langle U_{r}+u G(\mu),(L-\mu) b(L) V\right\rangle_{H}+\langle\vec{u},(\mathcal{M}-\mu) \Gamma \vec{v}\rangle_{\mathbb{C}^{m}} .
$$

It follows that the action of $\mathbb{A}_{\min }$ is given by formula (5.10).

Note that $\mathbb{A}_{\text {min }}$ is an extension of $L_{\text {min }}$.

It is easy to see that $\mathbb{A}_{\min }$ is symmetric in $\mathbb{H}$ or, in other words, is a restriction of $\mathbb{A}_{\max }$, if and only if the matrices $\mathcal{M}$ and $\Gamma$ commute

$$
\Gamma \mathcal{M}=\mathcal{M} \Gamma .
$$

Here $\mathcal{M}$ is diagonal with all diagonal elements pairwise different while $\Gamma$ is Hermitian and positive definite. Hence, in order to satisfy (5.12), $\Gamma$ has to be diagonal as well, and all diagonal elements must be positive numbers. Therefore, in the sequel we assume that $\Gamma$ is diagonal and positive definite. Under this assumption, (5.10) takes the form

$$
\mathbb{A}_{\min }\left(\begin{array}{c}
U_{r} \\
\vec{u}
\end{array}\right)=\left(\begin{array}{c}
L U_{r} \\
\mathcal{M} \vec{u}
\end{array}\right) .
$$

Now we are in the situation described by (4.2), and all self-adjoint restrictions of $\mathbb{A}_{\max }$ can be obtained using classical Birman-Krein-Vishik extension theory for symmetric operators in a Hilbert space. 


\section{The self-adjoint family of extensions}

In this section, we calculate explicitly the one-parameter family of self-adjoint operators in $\mathbb{H}$ satisfying (4.3) with $\mathbb{A}_{\min }, \mathbb{A}_{\max }$ from the previous section. One particular extension, denoted by $\mathbb{A}_{0}$, is not hard to guess:

$$
\mathbb{A}_{0}=L \oplus \mathcal{M}, \quad \operatorname{Dom}\left(\mathbb{A}_{0}\right)=\mathcal{H}_{m+2} \oplus \mathbb{C}^{n-2} .
$$

Let us calculate the defect and deficiency element for the symmetric operator $\mathbb{A}_{\text {min }}$. The operator $\mathbb{A}_{\max }$ is closed and therefore is the adjoint operator to $\mathbb{A}_{\min }$. Any deficiency element $\mathbb{F}(\lambda), \Im \lambda \neq 0$, is a nontrivial solution to the equation

$$
\left(\mathbb{A}_{\max }-\lambda\right) \mathbb{F}(\lambda)=0
$$

which can be written in the form

$$
\left\{\begin{array}{l}
(L-\lambda) F_{r}+(\mu-\lambda) G(\mu) f=0 \\
(\mathcal{M}-\lambda) \vec{f}+f \vec{b}=0
\end{array}\right.
$$

Any solution to this system is a multiple of

$$
\mathbb{F}(\lambda)=\left(\begin{array}{c}
\frac{L-\mu}{L-\lambda} G(\mu) \\
-\frac{1}{\mathcal{M}-\lambda} \vec{b}
\end{array}\right)=\left(\begin{array}{c}
G(\lambda) \\
-\frac{1}{\mathcal{M}-\lambda} \vec{b}
\end{array}\right)
$$

We conclude that $\mathbb{A}_{\text {min }}$ has deficiency indices $(1,1)$. With this parametrization, the family of deficiency elements has the important property

$$
\mathbb{F}(z)=\frac{\mathbb{A}_{0}-\lambda}{\mathbb{A}_{0}-z} \mathbb{F}(\lambda)
$$

Every element $\mathbb{F}(\lambda)$ can also be viewed as a function from $\mathcal{H}_{m}$

$$
\mathbb{F}(\lambda)=\frac{1}{b(\lambda)} g(\lambda)
$$

To prove this, one can use the formula

$$
\frac{1}{b(L)}=\sum_{j=1}^{n-2} \frac{1}{b_{j}\left(\mu_{j}\right)} \frac{1}{L-\mu_{j}}
$$


with $b_{j}$ given by (4.11), and its natural modification

$$
\begin{aligned}
\mathbb{F}(\lambda) & =G(\lambda)-\sum_{j=1}^{m} \frac{1}{\mu_{j}-\lambda} \frac{1}{b_{j}\left(\mu_{j}\right)} g\left(\mu_{j}\right)=\frac{1}{b(L)} g(\lambda)+\sum_{j=1}^{m} \frac{1}{b_{j}\left(\mu_{j}\right)} \frac{1}{\lambda-\mu_{j}} g\left(\mu_{j}\right) \\
& =\sum_{j=1}^{m}\left(\frac{1}{b_{j}\left(\mu_{j}\right)} \frac{1}{L-\mu_{j}} g(\lambda)+\frac{1}{b_{j}\left(\mu_{j}\right)} \frac{1}{\lambda-\mu_{j}} g\left(\mu_{j}\right)\right) \\
& =\sum_{j=1}^{m} \frac{1}{b_{j}\left(\mu_{j}\right)} \frac{1}{\lambda-\mu_{j}} g(\lambda) \\
& =\frac{1}{b(\lambda)} g(\lambda) .
\end{aligned}
$$

It follows that the functions $g(\lambda)$ (multiplied by a scalar factor) play the role of deficiency elements in our construction.

Now it is standard to determine the family of self-adjoint extensions of the minimal operator $\mathbb{A}_{\min }$, which are at the same moment restrictions of the maximal operator $\mathbb{A}_{\max }$. All such restrictions and their resolvents are described by Theorem 6.1 below.

Such operators can always be determined by certain boundary conditions connecting the "boundary values"

$$
u, \vec{u} \quad \text { and }\left\langle g(\mu),(L-\mu) U_{r}\right\rangle_{H} .
$$

Under the condition that $\Gamma$ is diagonal, the boundary form of $\mathbb{A}_{\max }$ is (see (5.8))

(6.5) $\left\langle\mathbb{A}_{\max } \mathbb{U}, \mathbb{V}\right\rangle_{\mathbb{H}}-\left\langle\mathbb{U}, \mathbb{A}_{\max } \mathbb{V}\right\rangle_{\mathbb{H}}$

$$
=\bar{u}\left(\langle\Gamma \vec{b}, \vec{v}\rangle_{\mathbb{C}^{n-2}}-\left\langle g(\mu),(L-\mu) V_{r}\right\rangle_{H}\right)-\overline{\left(\langle\Gamma \vec{b}, \vec{u}\rangle_{\mathbb{C}^{n-2}}-\left\langle g(\mu),(L-\mu) U_{r}\right\rangle_{H}\right)} v .
$$

We define then the following restrictions of the maximal operator and show that these are exactly the self-adjoint extensions.

Definition 6.1. The domain of the operator $\mathbb{A}_{\theta}, \theta \in[0, \pi)$, consists of functions $\mathbb{U} \in \mathbb{H}$ possessing the representation

$$
\mathbb{U}=\left(\begin{array}{l}
U \\
\vec{u}
\end{array}\right)=\left(\begin{array}{c}
U_{r}+u G(\mu) \\
\vec{u}
\end{array}\right), \quad U_{r} \in \mathcal{H}_{n}, u \in \mathbb{C}, \vec{u} \in \mathbb{C}^{n-2},
$$

and satisfying the boundary condition

$$
\sin \theta\left\langle g(\mu),(L-\mu) U_{r}\right\rangle_{H}+\cos \theta u-\sin \theta\langle\Gamma \vec{b}, \vec{u}\rangle_{\mathbb{C}^{n-2}}=0 .
$$

The action of $\mathbb{A}_{\theta}$ is given by the formula

$$
\mathbb{A}_{\theta} \mathbb{U}=\mathbb{A}_{\theta}\left(\begin{array}{c}
U_{r}+u G(\mu) \\
\vec{u}
\end{array}\right)=\left(\begin{array}{c}
L U_{r}+\mu u G(\mu) \\
\mathcal{M} \vec{u}+u \vec{b}
\end{array}\right),
$$


where the matrix $\mathcal{M}$ and the vector $\vec{b}$ are determined by (5.3) and (5.4).

Note that the operator $\mathbb{A}_{0}$ introduced earlier coincides with $\mathbb{A}_{\theta}$ for $\theta=0$. It is straightforward to calculate the resolvent of $\mathbb{A}_{\theta}$.

Theorem 6.1. Let $\Gamma$ be a positive diagonal matrix. Then the family of selfadjoint restrictions of the maximal operator $\mathbb{A}_{\max }$ coincides with the family $\mathbb{A}_{\theta}, \theta \in$ $[0, \pi)$. The resolvent of the operator $\mathbb{A}_{\theta}$ for $\Im \lambda \neq 0$ is given by

$$
\begin{aligned}
& \frac{1}{\mathbb{A}_{\theta}-\lambda}-\frac{1}{\mathbb{A}_{0}-\lambda} \\
& =-\frac{1}{Q(\lambda)+\cot \theta}\left(\begin{array}{cc}
\langle g(\bar{\lambda}), \cdot\rangle_{H} G(\lambda) & \left\langle\frac{-1}{\mathcal{M}-\lambda} \vec{b}, \Gamma \cdot\right\rangle_{\mathbb{C}^{m}} G(\lambda) \\
\langle g(\bar{\lambda}), \cdot\rangle_{H} \frac{-1}{\mathcal{M}-\lambda} \vec{b} & \left\langle\frac{-1}{\mathcal{M}-\lambda} \vec{b}, \Gamma \cdot\right\rangle_{\mathbb{C}^{m}} \frac{-1}{\mathcal{M}-\lambda} \vec{b}
\end{array}\right),
\end{aligned}
$$

where

$$
Q(\lambda)=\langle g(\mu),(\lambda-\mu) G(\lambda)\rangle_{H}+\left\langle\vec{b}, \Gamma \frac{1}{\mathcal{M}-\lambda} \vec{b}\right\rangle_{\mathbb{C}^{m}}
$$

Proof. Since the matrix $\Gamma$ is diagonal, the boundary form of the maximal operator is given by (6.5); and it is then clear that the restriction of $\mathbb{A}_{\max }$ to the set of functions satisfying (6.7) is a symmetric operator (the boundary form vanishes).

Let us calculate directly the resolvent of $\mathbb{A}_{\theta}$. Consider the resolvent equation for $\lambda \in \mathbb{C} \backslash \mathbb{R}$,

$$
\frac{1}{\mathbb{A}_{\theta}-\lambda} \mathbb{V}=\mathbb{U} \Rightarrow \mathbb{V}=\left(\mathbb{A}_{\theta}-\lambda\right) \mathbb{U}
$$

where $\mathbb{V} \in \mathbb{H}$ and $\mathbb{U} \in \operatorname{Dom}\left(\mathbb{A}_{\theta}\right)$. The last equation implies

$$
\begin{gathered}
\left\{\begin{array}{l}
V=(L-\lambda) U_{r}+(\mu-\lambda) G(\mu) u, \\
\vec{v}=(\mathcal{M}-\lambda) \vec{u}+\vec{b} u
\end{array}\right. \\
\Rightarrow\left\{\begin{array}{l}
U_{r}=\frac{1}{L-\lambda} V-\frac{\mu-\lambda}{L-\lambda} G(\mu) u \\
\vec{u}=\frac{1}{\mathcal{M}-\lambda} \vec{v}-\frac{1}{\mathcal{M}-\lambda} \vec{b} u .
\end{array}\right.
\end{gathered}
$$

Substituting into the boundary condition (6.7), we calculate

$$
u=\frac{-\langle g(\bar{\lambda}), V\rangle_{H}+\left\langle\Gamma \vec{b}, \frac{1}{\mathcal{M}-\lambda} \vec{v}\right\rangle_{\mathbb{C}^{m}}}{\langle g(\mu),(\lambda-\mu) G(\lambda)\rangle_{H}+\left\langle\vec{b}, \Gamma \frac{1}{\mathcal{M}-\lambda} \vec{b}\right\rangle_{\mathbb{C}^{m}}+\cot \theta} .
$$

It is natural to denote the Nevanlinna function appearing in the denominator by $Q(\lambda)$ (see (6.10). Then all components of the function $\mathbb{U}$ can be calculated

$$
\left\{\begin{array}{l}
U=\frac{1}{L-\lambda} V-\frac{1}{Q(\lambda)+\cot \theta}\left\{\langle g(\bar{\lambda}), V\rangle_{H} G(\mu)+\left\langle\frac{-1}{\mathcal{M}-\lambda} \vec{b}, \Gamma \vec{v}\right\rangle_{\mathbb{C}^{n-2}} G(\mu)\right\}, \\
\vec{u}=\frac{1}{\mathcal{M}-\lambda} \vec{v}-\frac{1}{Q(\lambda)+\cot \theta}\left\{\langle g(\lambda), V\rangle_{H} \frac{-1}{\mathcal{M}-\lambda} \vec{b}+\left\langle\frac{-1}{\mathcal{M}-\lambda} \vec{b}, \Gamma \vec{v}\right\rangle_{\mathbb{C}^{n-2}} \frac{-1}{\mathcal{M}-\lambda} \vec{b}\right\}
\end{array}\right.
$$


which implies formula (6.9). Hence every operator $\mathbb{A}_{\theta}$ is symmetric, and the range of $\mathbb{A}_{\theta}-\lambda$, $\Im \lambda \neq 0$, coincides with all of $\mathbb{H}$. Thus every such operator is self-adjoint.

On the other, hand formula (6.9) can be written in Krein's form as

$$
\frac{1}{\mathbb{A}_{\theta}-\lambda}-\frac{1}{\mathbb{A}_{0}-\lambda}=-\frac{1}{Q(\lambda)+\cot \theta}\langle\mathbb{F}(\bar{\lambda}), \cdot\rangle_{\mathbb{H}} \mathbb{F}(\lambda)
$$

where $\mathbb{F}(\lambda)$ is given by (6.1). This proves that the family $\mathbb{A}_{\theta}$ is indeed the family of all possible self-adjoint restrictions of $\mathbb{A}_{\max }$.

The resolvent formulas just proved $(6.9,6.11)$ are classical Krein formulas, and the $Q$-function appearing in the denominator is a Nevanlinna function, since the operators $\mathbb{A}_{0}, \mathbb{A}_{\theta}$ are self-adjoint in the Hilbert space $\mathbb{H}$. In the following subsection, we present another resolvent formula associated with the particular structure of the triplet extensions.

\section{Extended resolvent formula of Krein type}

The Hilbert space $\mathbb{H}$ decomposes naturally into the orthogonal sum of the infinite dimensional space $\mathcal{H}_{m}$ and a finite dimensional space $\mathbb{C}^{m}$ in accordance to (4.14). It is clear therefore that the compression of the resolvent to the infinite dimensional component is given by Krein's formula for generalized resolvents with the denominator equal to a sum of two Nevanlinna functions:

$$
\begin{aligned}
\left.P_{\mathcal{H}_{m}} \frac{1}{\mathbb{A}_{\theta}-\lambda}\right|_{\mathcal{H}_{m}} & =\frac{1}{L-\lambda}-\frac{1}{Q(\lambda)+\cot \theta}\langle g(\bar{\lambda}), \cdot\rangle_{H} G(\lambda) \\
& =\frac{1}{L-\lambda}-\frac{1}{q(\lambda)+q_{\Gamma}(\lambda)+\cot \theta}\langle G(\bar{\lambda}), \cdot\rangle_{\mathcal{H}_{m}} G(\lambda),
\end{aligned}
$$

where

- $q(\lambda)=\langle g(\mu),(\lambda-\mu) G(\lambda)\rangle_{H}$ is the $Q$-function associated with the operators $L$ and $L_{\min }$ in $\mathcal{H}_{m}$, and

- $q_{\Gamma}(\lambda)=\left\langle\vec{b}, \Gamma \frac{1}{\mathcal{M}-\lambda} \vec{b}\right\rangle_{\mathbb{C}^{m}}$ is the $Q$-function associated with the operator $\mathcal{M}$ and vector $\vec{b}$ in $\mathbb{C}^{m}$.

This resolvent formula shows once again that the operator $\mathbb{A}_{\theta}$ is indeed a generalized extension of the operator $L_{\min }$.

Let us now consider another type of resolvent formula - the re striction of the resolvent of $\mathbb{A}_{\theta}$ to $\mathcal{H}_{m}$, but written in the functional representation

$$
\begin{aligned}
\left.\frac{1}{\mathbb{A}_{\theta}-\lambda}\right|_{\mathcal{H}_{m}} & =\frac{1}{L-\lambda}-\frac{1}{Q(\lambda)+\cot \theta}\langle g(\bar{\lambda}), \cdot\rangle_{H} \mathbb{F}(\lambda) \\
& =\frac{1}{L-\lambda}-\frac{1}{b(\lambda)(Q(\lambda)+\cot \theta)}\langle g(\bar{\lambda}), \cdot\rangle_{H} g(\lambda)
\end{aligned}
$$


The function appearing in the denominator

$$
\begin{aligned}
Q_{m}(\lambda) & =b(\lambda)(Q(\lambda)+\cot \theta) \\
& =b(\lambda)\left(\left\langle\varphi, \frac{1}{L-\lambda} \frac{\lambda-\mu}{L-\mu} \frac{1}{b(L)} \varphi\right\rangle_{H}+\left\langle\vec{b}, \Gamma \frac{1}{\mathcal{M}-\lambda} \vec{b}\right\rangle_{\mathbb{C}^{n-2}}+\cot \theta\right)
\end{aligned}
$$

is a generalized Nevanlinna function (see [13]). In the following section, we show that this function can be obtained by regularizing the classical formula (2.12), valid for canonical extensions.

We emphasize that a generalized Nevanlinna function appears in our model despite the fact that only self-adjoint operators in Hilbert space are involved.

\section{Renormalization of the $Q$-function}

For canonical extensions (inside the original Hilbert space), the functions $g(\lambda)$ and $G(\lambda)$ coincide, since this case corresponds to $m=0$ and $b \equiv 1$. The function (2.12) appearing in the denominator of Krein's formula in the case of canonical extensions can be considered as a renormalization of the Nevanlinna function

$$
Q(\lambda)=\left\langle g(\mu), \frac{(L-\mu)^{2}}{L-\lambda} g(\mu)\right\rangle_{H}=\langle g(\mu),(L-\mu) g(\lambda)\rangle_{H},
$$

which is well-defined only if $g(\mu) \in \mathcal{H}_{1}$. In fact, precisely this function appears in the resolvent formula when the perturbed operator is a bounded rank one perturbation (see [5] and Section 9).

If $g(\mu) \in \mathcal{H} \backslash \mathcal{H}_{1}$, then the function $Q$ can be obtained using the following renormalization procedure;

$$
\begin{aligned}
Q(\lambda) & \stackrel{\text { formally }}{=}\left\langle g(\mu), \frac{(L-\mu)^{2}}{L-\lambda} g(\mu)\right\rangle_{H}-\langle g(\mu),(L-\mu) g(\mu)\rangle_{H}+p \\
& =\langle g(\mu),(\lambda-\mu) g(\lambda)\rangle_{H}+p
\end{aligned}
$$

with the renormalization point $\mu<0$ and renormalization parameter $p \stackrel{\text { formally }}{=}$ $\langle g(\mu),(L-\mu) g(\mu)\rangle_{H} \in \mathbb{R}$. If $g(\mu) \in \mathcal{H}_{1}$, then the renormalization parameter is uniquely determined by the last formula; if $g(\mu) \in \mathcal{H} \backslash \mathcal{H}_{1}$, then this parameter can be chosen arbitrarily. ${ }^{3}$

This renormalization procedure can be continued in order to include more and more singular elements $g(\mu)$. For example, if $g(\mu) \in \mathcal{H}_{-1} \backslash \mathcal{H}$, then the scalar product $\langle g(\mu), g(\lambda)\rangle_{H}$ is not defined and one needs one further renormalization

\footnotetext{
${ }^{3}$ This is connected with the fact that $\mathcal{H}_{-1}$-perturbations are uniquely determined, but $\mathcal{H}_{-2^{-}}$ perturbations not (see Section 9).
} 
with a certain $\mu_{1}<0$ and $p_{1} \in \mathbb{R}$,

$$
\begin{gathered}
Q_{1}(\lambda) \stackrel{\text { formally }}{=}(\lambda-\mu)\left\{\langle g(\mu), g(\lambda)\rangle_{H}-\left\langle g(\mu), g\left(\mu_{1}\right)\right\rangle_{H}+p_{1}\right\}+p \\
=\left\langle g(\mu),(\lambda-\mu) \frac{\lambda-\mu_{1}}{L-\mu_{1}} g(\lambda)\right\rangle_{H}+(\lambda-\mu) p_{1}+p .
\end{gathered}
$$

The renormalization parameter $p_{1}$ is formally equal to $\left\langle g(\mu), g\left(\mu_{1}\right)\right\rangle_{H}$; and if $g(\mu) \in \mathcal{H}$, then this parameter is uniquely defined and the $Q$-function coincides with the function given by (8.1). The function $Q_{1}$ contains two renormalization parameters $p$ and $p_{1}$ and is not necessarily a Nevanlinna function, but rather a generalized Nevanlinna function, with one negative square.

Continuing this renormalization procedure, we obtain the following formula for the $Q$-function in the case $g(\mu) \in \mathcal{H}_{-m} \backslash H_{-m+1}, m \geq 1$,

$$
\begin{aligned}
Q_{m}(\lambda) & =\left\langle g(\mu),(\lambda-\mu) \frac{\lambda-\mu_{1}}{L-\mu_{1}} \cdots \frac{\lambda-\mu_{m}}{L-\mu_{m}} g(\lambda)\right\rangle_{H}+p(\lambda) \\
& =\left\langle g(\mu),(\lambda-\mu) \frac{b(\lambda)}{b(L)} g(\lambda)\right\rangle_{H}+p(\lambda)
\end{aligned}
$$

where

$$
p(\lambda)=(\lambda-\mu) \sum_{j=1}^{m}\left(\lambda-\mu_{1}\right) \cdots\left(\lambda-\mu_{j-1}\right) p_{j}+p .
$$

The renormalization points $\mu_{j}$ are all chosen negative $\mu_{j}<0$, and the real renormalization parameters are formally equal to the scalar products

$$
p_{j} \stackrel{\text { formally }}{=}\left\langle g(\mu), \frac{1}{\left(L-\mu_{j}\right)\left(L-\mu_{j-1}\right) \cdots\left(L-\mu_{2}\right)} g\left(\mu_{1}\right)\right\rangle_{H} .
$$

As before, this sequence of $Q$-functions is constructed in such a way that if the deficiency element is less singular, so that some of the scalar products in (8.5) are well-defined, then the renormalization parameters $p_{j}$ can be properly chosen to get the $Q$-function corresponding to the less singular deficiency elements.

The function $Q_{m}$ is a generalized Nevanlinna function with at most $\left[\frac{m+1}{2}\right]$ negative squares and contains $m+1$ arbitrary real parameters $p, p_{j}$. If the deficiency elements are less singular, for example if $g(\mu) \in \mathcal{H}_{-l}, l<m-1$, then the parameters can be chosen in such a way that the $Q$-function has fewer negative squares. In particular, if $g(\mu) \in \mathcal{H}$, then the function $Q_{m}$ can be made a Nevanlinna function by choosing the renormalization parameters properly. Moreover, if $g(\mu) \in \mathcal{H}_{-m} \backslash \mathcal{H}$, then $Q_{m}$ is not a usual Nevanlinna function, independently of how the parameters $p, p_{j}$ are chosen. 
Formulas (8.3) and (7.3) give the same function if and only if the polynomial $p(\lambda)$ is chosen to be

$$
p(\lambda)=-\sum_{j=1}^{m} \frac{\gamma_{j j}^{2}}{\left(b_{j}\left(\mu_{j}\right)\right)^{2}} b_{j}(\lambda)+b(\lambda) \cot \theta,
$$

where $b_{j}$ are given by (4.11) and $\gamma_{j j}$ are the entries of $\Gamma$.

\section{Triplet extensions and supersingular perturbations}

Let $L$ be a positive self-adjoint operator acting in the Hilbert space $H$. Let $\varphi$ be an element from the scale of Hilbert spaces $\mathcal{H}_{n}$ associated with the operator $L$,

$$
\varphi \in \mathcal{H}_{-n} \backslash \mathcal{H}_{-n+1}
$$

Then a rank one perturbation of the operator $L$ is given by the formal expression

$$
L+\alpha\langle\varphi,\rangle_{H} \varphi, \quad \alpha \in \mathbb{R}
$$

In this section, we construct self-adjoint operators corresponding to the formal expression (9.2) in the case $n>2$. Such perturbations are usually called supersingular, in order to distinguish them from the singular perturbations given by $\varphi \in \mathcal{H}_{-2} \backslash \mathcal{H}$.

Let us recall first the main ideas of the theory of regular $(n=0)$ and singular ( $n=1,2)$ perturbations. If $\varphi \in \mathcal{H}_{0}=H$, then the perturbation in (9.2) is bounded and the perturbed operator, denoted by $L_{\alpha}$, is self-adjoint on $\operatorname{Dom}(L)$. If $\varphi \in \mathcal{H}_{-1}$, then (9.2) determines a unique operator $L_{\alpha}$, since the perturbation is relative form bounded in this case and the method of quadratic forms can be applied. It is also possible to use the extension theory for symmetic operators and identify the operator given by (9.2) with one particular self-adjoint extension of the symmetric operator $L_{\min }=\left.L\right|_{\left\{U \in \operatorname{Dom}(L):\langle\varphi, U\rangle_{H}=0\right\}}$. If $\varphi \in \mathcal{H}_{-2} \backslash \mathcal{H}_{-1}$, then the quadratic form approach cannot be applied; but the extension theory approach can be used, since $L_{\text {min }}$ is well-defined as a symmetric (not essentially self-adjoint operator) in $H$. Then the operator corresponding to (9.2) is usually defined as an operator from the one-parameter family of self-adjoint extensions of $L_{\min }$. In general, it is impossible to decide which particular extension corresponds to formula (9.2), which is understood formally; in order to emphasize this, we denote the corresponding operator by $A_{\gamma}, \gamma \in \mathbb{R} \cup\{\infty\}$, instead of $L_{\alpha}$. Using the fact that the deficiency elements for the symmetric operator $L_{\min }$ are

$$
g(\lambda)=\frac{1}{L-\lambda} \varphi
$$


we can describe the resolvent of any operator $A_{\gamma}$ by Krein's formula

$$
\frac{1}{A_{\gamma}-\lambda}=\frac{1}{L-\lambda}-\frac{1}{Q(\lambda)+\gamma}\left\langle\frac{1}{L-\bar{\lambda}} \varphi, \cdot\right\rangle_{H} \frac{1}{L-\lambda} \varphi, \quad \Im \lambda \neq 0,
$$

where $Q(\lambda)$ is given by

$$
Q(\lambda)=\left\langle\varphi, \frac{\lambda-\mu}{(L-\lambda)(L-\mu)} \varphi\right\rangle_{H}+p
$$

and $\mu<0$ and $p \in \mathbb{R}$ are arbitrary parameters. The same formula (9.4) gives the resolvent of the operator $L_{\alpha}$ in the case of $\mathcal{H}_{-1}$-perturbations if the parameters are properly chosen as

$$
p=\left\langle\varphi, \frac{1}{L-\mu} \varphi\right\rangle_{H} \quad \text { and } \quad \gamma=1 / \alpha
$$

In this case, the $Q$-function is just equal to

$$
Q(\lambda)=\left\langle\varphi, \frac{1}{L-\lambda} \varphi\right\rangle_{H}
$$

It is precisely this renormalization procedure that was generalized in Section 8.

Summing up, to define singular perturbations given by (9.2), classical extension theory of symmetric operators may be used. The function $Q(\lambda)$ is a Nevanlinna function and contains information about spectral properties of the operator $A_{\gamma}$.

Let us discuss now supersingular perturbations given by vectors $\varphi \in$ $\mathcal{H}_{-n} \backslash \mathcal{H}_{-n+1}, n>2$. The formal expression (9.2) naturally leads to the minimal operator

$$
L_{\min }=\left.L\right|_{\left\{U \in \mathcal{H}_{n}:\langle\varphi, U\rangle_{H}=0\right\}} .
$$

This is a symmetric operator in the Hilbert space $\mathcal{H}_{n-2}$ and has deficiency indices $(1,1)$ if considered in this Hilbert space. It is clear that the functions $g(\lambda)$ and $G(\lambda)$ are given in this case by

$$
g(\lambda)=\frac{1}{L-\lambda} \varphi \in \mathcal{H}_{-n+2}, \quad G(\lambda)=\frac{1}{b(L)} \frac{1}{L-\lambda} \varphi \in \mathcal{H}_{n-2} .
$$

Since $\varphi \notin \mathcal{H}_{-2}, L_{\text {min }}$ is essentially self-adjoint in the original Hilbert space $H$ and therefore satisfies the assumptions formulated in Section 3 with $m=n-2$. Any self-adjoint operator associated with (9.2) must be an extension of $L_{\min }$, and it is natural to associate with it the family of triplet extensions constructed in Section 6. The kernel of the operator $L_{\max }-\lambda=L_{\min }^{\dagger}-\lambda$ is spanned by the functions $g(\lambda)$. The model space $\mathbb{H}$ is then given by

(9.9) $\mathbb{H}=\mathcal{H}_{n-2} \dot{+} \mathcal{L}\left\{\left(L-\mu_{1}\right)^{-1} \varphi,\left(L-\mu_{2}\right)^{-1} \varphi, \ldots,\left(L-\mu_{n-2}\right) \varphi\right\} \ni \mathbb{U}=(U, \vec{u})$ 
and endowed with the scalar product given by (4.12). The elements from the space can also be viewed as elements from $\mathcal{H}_{-n+2}$,

$$
\mathbb{U}=U+\sum_{j=1}^{n-2} u_{j}\left(L-\mu_{j}\right)^{-1} \varphi
$$

since $\left(L-\mu_{j}\right)^{-1} \varphi \in \mathcal{H}_{-n+2}$. Constructing the space $\mathbb{H}$, we have chosen $n-2$ arbitrary negative renormalization points $\mu_{j}<0, j=1,2, \ldots, n-2$, and $n-2$ arbitrary positive parameters $\gamma_{j j}>0, j=1,2, \ldots, n-2$, since the Gram matrix $\Gamma$ in (4.12) has to be chosen diagonal and positive in order to guarantee the existence of self-adjoint triplet extensions.

The one-parameter family $\mathbb{A}_{\theta}, \theta \in[0, \pi)$ of triplet extensions of $L_{\min }$ in $\mathbb{H}$ is then defined on the domain of functions possessing the representation

$$
\mathbb{U}=\left(U_{r}+u b^{-1}(L)(L-\mu)^{-1} \varphi, \vec{u}\right), \quad U_{r} \in \mathcal{H}_{n}, u \in \mathbb{C}, \vec{u} \in \mathbb{C}^{n-2}
$$

and satisfying the boundary condition

$$
\sin \theta\left\langle\varphi, U_{r}\right\rangle_{H}+\cos \theta u-\sin \theta\langle\Gamma \vec{b}, \vec{u}\rangle_{\mathbb{C}^{n-2}}=0 .
$$

Note that $b^{-1}(L)(L-\mu)^{-1} \varphi \in \mathcal{H}_{n-2}$. The action of the operator $\mathbb{A}_{\theta}$ is given by

$$
\mathbb{A}_{\theta} \mathbb{U}=\mathbb{A}_{\theta}\left(\begin{array}{c}
U_{r}+u b^{-1}(L)(L-\mu)^{-1} \varphi \\
\vec{u}
\end{array}\right)=\left(\begin{array}{c}
L U_{r}+\mu u b^{-1}(L)(L-\mu)^{-1} \varphi \\
\mathcal{M} \vec{u}+u \vec{b}
\end{array}\right)
$$

where the matrix $\mathcal{M}$ and the vector $\vec{b}$ are determined by (5.3) and (5.4). Taking into account (9.8), we can calculate the resolvent of the self-adjoint operator $\mathbb{A}_{\theta}$

$$
\begin{aligned}
& \frac{1}{\mathbb{A}_{\theta}-\lambda}-\frac{1}{\mathbb{A}_{0}-\lambda} \\
& =-\frac{1}{Q(\lambda)+\cot \theta}\left(\begin{array}{cc}
\left\langle\frac{1}{L-\lambda} \varphi, \cdot\right\rangle_{H} \frac{1}{b(L)(L-\lambda)} \varphi & \left\langle\frac{-1}{\mathcal{M}-\lambda} \vec{b}, \Gamma \cdot\right\rangle_{\mathbb{C}^{n-2}} \frac{1}{b(L)(L-\lambda)} \varphi \\
\left\langle\frac{1}{L-\lambda} \varphi, \cdot\right\rangle_{H} \frac{-1}{\mathcal{M}-\lambda} \vec{b} & \left\langle\frac{-1}{\mathcal{M}-\lambda} \vec{b}, \Gamma \cdot\right\rangle_{\mathbb{C}^{n-2}} \frac{-1}{\mathcal{M}-\lambda} \vec{b}
\end{array}\right),
\end{aligned}
$$

with

$$
Q(\lambda)=\left\langle\varphi, \frac{1}{b(L)} \frac{\lambda-\mu}{(L-\lambda)(L-\mu)} \varphi\right\rangle_{H}+\left\langle\vec{b}, \Gamma \frac{1}{\mathcal{M}-\lambda} \vec{b}\right\rangle_{\mathbb{C}^{n-2}}
$$

The extended resolvent formula (7.2) takes the form

$$
\left.\frac{1}{\mathbb{A}_{\theta}-\lambda}\right|_{\mathcal{H}_{n-2}}=\frac{1}{L-\lambda}-\frac{1}{b(\lambda)(Q(\lambda)+\cot \theta)}\left\langle\frac{1}{L-\bar{\lambda}} \varphi, \cdot\right\rangle_{H} \frac{1}{L-\lambda} \varphi,
$$

which is a natural generalization of (9.4). The denominator is given by the generalized Nevanlinna function

$$
Q_{n-2}(\lambda)=\left\langle\varphi, \frac{1}{L-\lambda} \frac{\lambda-\mu}{L-\mu} \frac{b(\lambda)}{b(L)} \varphi\right\rangle_{H}+p(\lambda)
$$


with the polynomial $p(\lambda)$ given by (8.6).

To summarize, supersingular perturbations, at least of semibounded operators, are given by triplet extensions. Their spectral properties are described by generalized Nevanlinna functions.

The peak model for supersingular perturbations presented here is a generalization of the cascade model described in $[22,12]$. The advantage of the new model is that the Gram matrix $\Gamma$ is now diagonal, which leads to simplification of all formulas and renders the study of the corresponding operator more transparent. In the new model, it is clear that all $\mu_{j}$ must be pairwise different, since otherwise the functions $\left(L-\mu_{j}\right) \varphi$ are not linearly independent.

\section{Point interactions in $\mathbb{R}^{3}$ : new family}

In this section, we apply methods developed in the previous section to construct a new family of point interactions in $\mathbb{R}^{3} \ni \mathbf{x}=\left(x_{1}, x_{2}, x_{3}\right)$. Classical point interaction goes back to E. Fermi [16]; in three-dimensional space, it can be described formally by

$$
-\Delta+\alpha \delta=-\Delta+\alpha \delta\langle\delta, \cdot\rangle
$$

where $\delta$ is Dirac's delta function in $\mathbb{R}^{3}$ and $\alpha \in \mathbb{R} \cup\{\infty\}$ is a coupling constant. F. A. Berezin and L. D. Faddeev [7] suggested interpreting this operator using a restriction-extension procedure. This procedure can be summarized as follows. Consider the restriction of the Laplace operator to the set of functions in $W_{2}^{2}\left(\mathbb{R}^{3}\right)$ which vanish at the origin. ${ }^{4}$ Note that the perturbation term vanishes on such functions; therefore, the Laplace operator and any self-adjoint operator corresponding to (10.1) are two (different) extensions of the restricted operator. This construction provides a rigorous mathematical foundation for the celebrated Fermi pseudopotential [16], widely used in physics and chemistry [11]. The most serious limitation of this method is connected with the fact that the deficiency element $g(\mu)=e^{i \sqrt{\mu}|\mathbf{x}|} / 4 \pi|\mathbf{x}|$ is spherically symmetric; therefore, the original Laplace operator and its perturbation given by (10.1) differ only on the subspace of spherically symmetric functions, i.e., this method allows one to introduce interaction in the $s$-channel only. The perturbed operator is defined on the set of functions $U=\tilde{U}+u g(\mu), \tilde{U} \in W_{2}^{2}\left(\mathbb{R}^{3}\right), u \in \mathbb{C}$, whose asymptotics at the origin are given by

$$
U(\mathbf{x})=\frac{u_{-}}{4 \pi|\mathbf{x}|}+u_{0}+o(1), \quad \mathbf{x} \rightarrow 0
$$

\footnotetext{
${ }^{4}$ This restriction is possible due to Sobolev embedding theorem.
} 
where $u_{-}, u_{0} \in \mathbb{C}$ can be considered as certain boundary values of the function $U$. More precisely, every self-adjoint operator corresponding to the formal expression (10.1) coincides with the (differential) Laplace operator $-\Delta$ defined on the domain of functions in $W_{2}^{2}\left(\mathbb{R}^{3} \backslash\{0\}\right)$ satisfying the boundary condition

$$
u_{0}=\gamma u_{-}, \quad \gamma \in \mathbb{R} \cup\{\infty\}
$$

Without any additional assumption, it is not possible to establish the connection between the real parameters $\alpha$ in (10.1) and $\gamma$ in (10.3) (except for the fact that $\alpha=0$ should correspond to $\gamma=\infty$ ), since this perturbation is from the class $\mathcal{H}_{-2}: \delta \in \mathcal{H}_{-2}(-\Delta) .^{5}$ This approach has been generalized to study numerous problems from mathematical physics and its applications [2, 11, 28, 29].

The limitations of the model described are connected first of all with the fact that the singular element determining the perturbation in (10.1) - Dirac's $\delta$-function — is spherically symmetric. Suppose we want to get similar mo dels where point interactions are not spherically symmetric. ${ }^{6}$ Intuitively, it is clear that one has to consider singular elements which are not spherically symmetric. Restricting our consideration to singular elements given by distributions, we take into account that any generalized function with support at the origin can be written as a linear combination of derivatives of the delta function. Here we consider only first order derivatives, which yields for the perturbed Laplacian the formal expression

$$
L_{\vec{\alpha}}=-\Delta+\sum_{i=1}^{3} \alpha^{i}\left\langle\partial_{x_{i}} \delta, \cdot\right\rangle \partial_{x_{i}} \delta,
$$

where $\alpha^{i}, i=1,2,3$ are real coupling constants and $\partial_{x_{i}}=\partial / \partial x_{i}$ denotes the derivative with respect to the variable $x_{i}$. The perturbation term is from the class $\mathcal{H}_{-3}$, since $\varphi_{j}=\partial_{x_{i}} \delta \in \mathcal{H}_{-3}(\Delta)$; it follows that in order to determine the operator $L_{\vec{\alpha}}$, the theory of supersingular perturbations must be applied (see previous Section). The rank of the perturbation is equal to three, but the elements $\varphi_{i}=\partial_{x_{i}} \delta, \quad i=1,2,3$ and the operator $-\Delta$ generate three mutually orthogonal subspaces. Thus the developed approach needs just a slight modification. Let us restrict our consideration to interactions commuting with the permutation of the coordinates, i.e. $\alpha_{1}=\alpha_{2}=\alpha_{3} \equiv \alpha$. We show later that the corresponding interaction is spherically symmetric but influences the $p$-channel (instead of the $s$-channel, as in the classical Fermi pseudopotential).

For the reader's convenience, we present here our model using function representation. Since the perturbation is from the class $\mathcal{H}_{-3}$, one needs to consider

\footnotetext{
${ }^{5}$ See Section 1.5 in [5] and [3], where such a relation is established using the homogeneity properties of the Laplace operator and the delta-function.

${ }^{6}$ Such models are needed to describe small objects having complicated geometry.
} 
only one renormalization point $\mu_{1}=-\beta_{1}^{2}, \beta_{1}>0$. The functions $g(\lambda)$ and $G(\lambda)$ can easily be calculated using (9.8) and the fact that $\frac{e^{i k|\times|}}{4 \pi|\mathbf{x}|}, k=\sqrt{\lambda}$ is the Green's function for the Laplacian:

$$
\begin{array}{rlr}
g_{j}(\lambda) & =\frac{\partial}{\partial x_{j}} \frac{e^{i k|\mathbf{x}|}}{4 \pi|\mathbf{x}|}=\frac{i k|\mathbf{x}|-1}{4 \pi|\mathbf{x}|^{3}} e^{i k|\mathbf{x}|} x_{j}, \quad j=1,2,3 \\
G_{i}(\lambda) & =\frac{1}{\beta_{1}^{2}+k^{2}}\left(g_{i}(\lambda)-g_{i}\left(-\beta_{1}^{2}\right)\right) & \\
& =\frac{1}{\beta_{1}^{2}+k^{2}} \frac{(i k|\mathbf{x}|-1) e^{i k|\mathbf{x}|}+\left(\beta_{1}|\mathbf{x}|+1\right) e^{-\beta_{1}|\mathbf{x}|}}{4 \pi|\mathbf{x}|^{3}} x_{i} .
\end{array}
$$

The functions $g_{i}(\lambda)$ are pairwise orthogonal in $W_{1}^{-1}\left(\mathbb{R}^{3}\right)=\mathcal{H}_{-1}(-\Delta)$ (just as functions having different symmetries), as well as the functions $G_{i}(\lambda)$.

Writing

$$
h(k, r)=\frac{i k r-1}{4 \pi r^{3}} e^{i k r}
$$

allows us to simplify the formulas of (10.5) to

$$
g_{j}(\lambda)=h(k,|\mathbf{x}|) x_{j} \quad \text { and } \quad G_{j}(\lambda)=\frac{h(k,|\mathbf{x}|)-h\left(i \beta_{1},|\mathbf{x}|\right)}{\beta_{1}^{2}+k^{2}} x_{j},
$$

keeping in mind that the function $h$ is just a combination of elementary functions.

The model Hilbert space can now be chosen equal to (10.7)

$$
\mathbb{H}=W_{2}^{1}\left(\mathbb{R}^{3}\right) \dot{+} \mathcal{L}\left\{g_{1}\left(-\beta_{1}^{2}\right), g_{2}\left(-\beta_{1}^{2}\right), g_{3}\left(-\beta_{1}^{2}\right)\right\} \ni \mathbb{U}=U+\sum_{i=1}^{3} u_{1}^{i} h\left(i \beta_{1},|\mathbf{x}|\right) x_{i} .
$$

Thus, every function from $\mathbb{H}$ possesses the representation

$$
\mathbb{U}=U+\sum_{i=1}^{3} u_{1}^{i} \frac{-\beta_{1}|\mathbf{x}|-1}{4 \pi|\mathbf{x}|^{3}} e^{-\beta_{1}|\mathbf{x}|} x_{i} \equiv U-\frac{\beta_{1}|\mathbf{x}|+1}{4 \pi|\mathbf{x}|^{3}} e^{-\beta_{1}|\mathbf{x}|} \mathbf{x}^{t} \mathbf{u}_{1},
$$

where $\mathbf{u}_{1}=\left(u_{1}^{1}, u_{1}^{2}, u_{1}^{3}\right)^{t} \in \mathbb{C}^{3}$ and $\mathbf{x}=\left(x_{1}, x_{2}, x_{3}\right)^{t} \in \mathbb{R}^{3}$, so that

$$
\mathbf{x}^{t} \mathbf{u}_{1}=x_{1} u_{1}^{1}+x_{2} u_{1}^{2}+x_{3} u_{1}^{3} .
$$

In (10.8), $U \in W_{2}^{1}\left(\mathbb{R}^{3}\right)$; therefore, this representation is unique. Hence, every element from $\mathbb{H}$ can be viewed not only as a function on $\mathbb{R}^{3}$ but also, via vector representation, as a pair $\mathbb{U}=\left(U, \mathbf{u}_{1}\right), U \in W_{2}^{1}\left(\mathbb{R}^{3}\right), \mathbf{u}_{1} \in \mathbb{C}^{3}$.

The norm in $\mathbb{H}$ can be chosen to be

$$
\|\mathbb{U}\|_{\mathbb{H}}^{2}=\left\|U+\sum_{i=1}^{3} u_{1}^{i} g_{i}\left(-\beta_{1}^{2}\right)\right\|_{\mathbb{H}}^{2}=\|U\|_{W_{2}^{1}\left(\mathbb{R}^{3}\right)}^{2}+\gamma\left\|\mathbf{u}_{1}\right\|^{2},
$$


where $\gamma$ is an arbitrary positive parameter.

To define the self-adjoint operator in $\mathbb{H}$ corresponding to the formal expression (10.4), consider another negative parameter $\mu=-\beta^{2}, \beta>0$ and the extension parameter $\theta \in[0, \pi)$. Then the operator $\mathbb{A}_{\theta}$ is defined on the set of functions possessing the representation

$$
\begin{aligned}
\mathbb{U} & =U_{r}+\sum_{i=1}^{3} u^{i} G_{i}\left(-\beta^{2}\right)+\sum_{i=1}^{3} u_{1}^{i} g_{i}\left(-\beta_{1}^{2}\right), \\
& =U_{r}+\frac{-(1+\beta|\mathbf{x}|) e^{-\beta|\mathbf{x}|}+\left(1+\beta_{1}|\mathbf{x}|\right) e^{-\beta_{1}|\mathbf{x}|}}{\left(\beta_{1}^{2}-\beta^{2}\right) 4 \pi|\mathbf{x}|^{3}} \mathbf{x}^{t} \mathbf{u}-\frac{1+\beta_{1}|\mathbf{x}|}{4 \pi|\mathbf{x}|^{3}} e^{-\beta_{1}|\mathbf{x}|} \mathbf{x}^{t} \mathbf{u}_{1}, \\
& =U_{r}+\frac{h(i \beta,|\mathbf{x}|)-h\left(i \beta_{1},|\mathbf{x}|\right)}{\beta_{1}^{2}-\beta^{2}} \mathbf{x}^{t} \mathbf{u}+h\left(i \beta_{1},|\mathbf{x}|\right) \mathbf{x}^{t} \mathbf{u}_{1}, \\
& U_{r} \in W_{2}^{3}\left(\mathbb{R}^{3}\right), \mathbf{u}, \mathbf{u}_{1} \in \mathbb{C}^{3},
\end{aligned}
$$

and the boundary conditions ${ }^{7}$

$$
\sin \theta\left\langle\partial_{i} \delta, U_{r}\right\rangle+\cos \theta u^{i}-\sin \theta \gamma u_{1}^{i}=0, \quad i=1,2,3 .
$$

The last condition can be written in vector notation as

$$
\sin \theta\left(\nabla U_{r}(0)+\gamma \mathbf{u}_{1}\right)=\cos \theta \mathbf{u} .
$$

The action of the operator $\mathbb{A}_{\theta}$ is determined by the formula

$$
\begin{gathered}
\mathbb{A}_{\theta}\left(U_{r}+\frac{-(1+\beta|\mathbf{x}|) e^{-\beta|\mathbf{x}|}+\left(1+\beta_{1}|\mathbf{x}|\right) e^{-\beta_{1}|\mathbf{x}|}}{\left(\beta_{1}^{2}-\beta^{2}\right) 4 \pi|\mathbf{x}|^{3}} \mathbf{x}^{t} \mathbf{u}-\frac{1+\beta_{1}|\mathbf{x}|}{4 \pi|\mathbf{x}|^{3}} e^{-\beta_{1}|\mathbf{x}|} \mathbf{x}^{t} \mathbf{u}_{1}\right) \\
=-\Delta U_{r}-\beta^{2} \frac{-(1+\beta|\mathbf{x}|) e^{-\beta|\mathbf{x}|}+\left(1+\beta_{1}|\mathbf{x}|\right) e^{-\beta_{1}|\mathbf{x}|}}{\left(\beta_{1}^{2}-\beta^{2}\right) 4 \pi|\mathbf{x}|^{3}} \mathbf{x}^{t} \mathbf{u} \\
-\frac{1+\beta_{1}|\mathbf{x}|}{4 \pi|\mathbf{x}|^{3}} e^{-\beta_{1}|\mathbf{x}|}\left(\mathbf{x}^{t} \mathbf{u}-\beta_{1}^{2} \mathbf{x}^{t} \mathbf{u}_{1}\right),
\end{gathered}
$$

which implies that outside the origin it acts pointwise just as the usual Laplacian

$$
\left(\mathbb{A}_{\theta} \mathbb{U}\right)(\mathbf{x})=-\left(\mathbb{U}_{x_{1} x_{1}}+\mathbb{U}_{x_{2} x_{2}}+\mathbb{U}_{x_{3} x_{3}}\right)(\mathbf{x}), \quad \mathbf{x} \neq 0 .
$$

Using (10.6), we can write the action of the operator as

$$
\begin{array}{r}
\mathbb{A}_{\theta}\left(U_{r}+\frac{h(i \beta,|\mathbf{x}|)-h\left(i \beta_{1},|\mathbf{x}|\right)}{\beta_{1}^{2}-\beta^{2}} \mathbf{x}^{t} \mathbf{u}+h\left(i \beta_{1},|\mathbf{x}|\right) \mathbf{x}^{t} \mathbf{u}_{1}\right) \\
=-\Delta U_{r}-\beta^{2} \frac{h(i \beta,|\mathbf{x}|)-h\left(i \beta_{1},|\mathbf{x}|\right)}{\beta_{1}^{2}-\beta^{2}} \mathbf{x}^{t} \mathbf{u}+h\left(i \beta_{1},|\mathbf{x}|\right)\left(\mathbf{x}^{t} \mathbf{u}-\beta_{1}^{2} \mathbf{x}^{t} \mathbf{u}_{1}\right),
\end{array}
$$

\footnotetext{
${ }^{7}$ In principle, it is possible to choose three different real extension parameters $\theta_{i}, i=1,2,3$, independently; but our aim is to construct a model operator corresponding to the formal expression (10.4) with all $\alpha_{i}$ equal, i.e., with the interaction commuting with permutations of the coordinates.
} 
As a result we obtain a spherically symmetric interaction, i.e., the corresponding operator commutes with rotations about the origin and reflections in planes passing through the origin. In particular, it commutes with permutations of the coordinates.

Lemma 10.1. The operator $\mathbb{A}_{\theta}, \theta \in[0, \pi)$, commutes with the rotations around the origin and reflections in planes passing through the origin.

Proof. To see this, it suffices to prove that the domain Dom $\left(\mathbb{A}_{\theta}\right)$ is invariant under the transformations considered, as we already know that the action of the operator is given by the Laplacian (10.14), which is invariant under rotations and reflections. The boundary condition for $\mathbb{A}_{\theta}$ can be written in the vector form (10.12). To prove that the domain of $\mathbb{A}_{\theta}$ is invariant under rotations we have to prove that every function $\mathbb{U}$ possessing representation (10.10) possesses the same representation after rotation and that every function satisfying the boundary condition (10.12) satisfies this condition after the rotation.

For $3 \times 3$ rotation matrix $\mathcal{R}$, define $\mathcal{R} F$ for the function $F=F(\mathbf{x}), \mathbf{x}=$ $\left(x_{1}, x_{2}, x_{3}\right)^{t} \in \mathbb{R}^{3}$, by

$$
(\mathcal{R} F)(\mathbf{x})=F\left(\mathcal{R}^{-1} \mathbf{x}\right)
$$

We first prove that the linear space $\mathbb{H}$ is invariant under rotations. It is clear that the subspace $W_{2}^{1}\left(\mathbb{R}^{3}\right)$ is invariant. Using the fact that $h(k,|\mathbf{x}|)$ is invariant under rotations, we see easily that

$$
\mathcal{R}\left(h\left(i \beta_{1},|\mathbf{x}|\right) \mathbf{x}^{t} \mathbf{u}_{1}\right)=h\left(i \beta_{1},|\mathbf{x}|\right)\left(\mathcal{R}^{-1} \mathbf{x}\right)^{t} \mathbf{u}_{1}=h(i \beta,|\mathbf{x}|) \mathbf{x}^{t}\left(\mathcal{R} \mathbf{u}_{1}\right) .
$$

Hence $\mathbb{H}$ is not only invariant under rotations but, in addition, any rotation around the origin in $\mathbb{R}^{3}$ corresponds to the rotation of the vector $\mathbf{u}_{1}$. The rotation matrix $\mathcal{R}$ induces a unitary transformation in $\mathbb{H}$.

Similarly, the set of functions possessing representation (10.10) is invariant under rotations, and rotation of the function $\mathbb{U}$ corresponds to rotations of the vector $\mathbf{u}_{1}$ and $\mathbf{u}$. Taking into account that

$$
\mathcal{R}(\nabla U(0))=(\nabla \mathcal{R} U)(0)
$$

we conclude that the boundary conditions (10.12) are preserved under rotations as well.

The proof for reflections in planes through the origin follows the same lines.

We emphasize that the operator $\mathbb{A}_{\theta}$ does not coincide with the classical point interaction Hamiltonian and is not even unitarily equivalent to it. We have obtained a new family of models which can easily be generalized to include higher derivatives and hence more and more singular interactions. 
The three $Q$-functions describing spectral properties of the model are all given by formula (9.14), which now takes the form

$$
Q^{i}(\lambda)=\left\langle\partial_{x_{i}} \delta, \frac{1}{L+\beta_{1}^{2}} \frac{\lambda+\beta^{2}}{(L-\lambda)\left(L+\beta^{2}\right)} \partial_{x_{i}} \delta\right\rangle_{L_{2}\left(\mathbb{R}^{3}\right)}+\frac{\gamma}{-\beta_{1}^{2}-\lambda}
$$

Taking into account that these functions are all equal, $Q^{1}(\lambda)=Q^{2}(\lambda)=Q^{3}(\lambda) \equiv$ $Q(\lambda)$, we can rewrite the last formula and calculate the function explicitly as

$$
\begin{aligned}
Q(\lambda) & =\frac{1}{3}\left\langle\delta, \frac{L}{L+\beta_{1}^{2}} \frac{\lambda+\beta^{2}}{(L-\lambda)\left(L+\beta^{2}\right)} \delta\right\rangle_{L_{2}\left(\mathbb{R}^{3}\right)}+\frac{\gamma}{-\beta_{1}^{2}-k^{2}} \\
& =\frac{1}{12 \pi}\left\{i k+\frac{\beta_{1}^{2}}{i k-\beta_{1}}+\beta+\frac{\beta_{1}^{2}}{\beta+\beta_{1}}\right\}+\frac{\gamma}{-\beta_{1}^{2}-k^{2}} .
\end{aligned}
$$

Then the resolvent formula (9.13) must be modified as follows

$$
\begin{aligned}
& \frac{1}{\mathbb{A}_{\theta}-\lambda}=\left(\begin{array}{cc}
\frac{1}{L-\lambda} & 0 \\
0 & \frac{1}{-\beta_{1}^{2}-\lambda}
\end{array}\right)-\frac{1}{Q(\lambda)+\cot \theta} \\
& \times \sum_{i=1}^{3}\left(\begin{array}{cc}
\left\langle\frac{1}{L-\lambda} \partial_{i} \delta, \cdot\right\rangle_{L_{2}\left(\mathbb{R}^{3}\right)} \frac{1}{\left(L+\beta_{1}^{2}\right)(L-\lambda)} \partial_{i} \delta & \frac{\gamma}{\beta_{1}^{2}+\lambda}\left\langle\mathbf{e}_{i}, \cdot\right\rangle_{\mathbb{C}^{3}} \frac{1}{\left(L+\beta_{1}^{2}\right)(L-\lambda)} \partial_{i} \delta \\
\left\langle\frac{1}{L-\lambda} \partial_{i} \delta, \cdot\right\rangle_{L_{2}\left(\mathbb{R}^{3}\right)} \frac{1}{\beta_{1}^{2}+\lambda} \mathbf{e}_{i} & \frac{\gamma}{\beta_{1}^{2}+\lambda}\left\langle\mathbf{e}_{i}, \cdot\right\rangle_{\mathbb{C}^{3} \frac{1}{\beta_{1}^{2}+\lambda} \mathbf{e}_{i}}
\end{array}\right),
\end{aligned}
$$

where $\mathbf{e}_{i} \in \mathbb{C}^{3}, i=1,2,3$, are standard basis vectors in $\mathbb{C}^{3}$ and the first term on the right hand side is the resolvent of $\mathbb{A}_{0}=L \oplus\left(-\beta_{1}^{2}\right)$. Using vector notation, we can write the resolvent as

(10.18)

$$
\begin{aligned}
\frac{1}{\mathbb{A}_{\theta}-\lambda}= & \left(\begin{array}{c}
\frac{1}{L-\lambda} \\
\frac{1}{-\beta_{1}^{2}-\lambda}
\end{array}\right)-\frac{1}{Q(\lambda)+\cot \theta} \\
& \times\left(\begin{array}{cc}
-\frac{h(k,|\mathbf{x}|)-h\left(i \beta_{1},|\mathbf{x}|\right)}{\beta_{1}^{2}+\lambda} \mathbf{x}^{t} \nabla\left(\frac{1}{-\Delta-\lambda} \cdot\right)(0) & \frac{\gamma}{\beta_{1}^{2}+\lambda} \frac{h(k,|\mathbf{x}|)-h\left(i \beta_{1},|\mathbf{x}|\right)}{\beta_{1}^{2}+\lambda} \mathbf{x}^{t} . \\
\frac{-1}{\beta_{1}^{2}+\lambda} \nabla\left(\frac{1}{L-\lambda} \cdot\right)(0) & \frac{\gamma}{\left(\beta_{1}^{2}+\lambda\right)^{2}} .
\end{array}\right) .
\end{aligned}
$$

The same formula can be written using the function representation as (10.19)

$$
\begin{aligned}
\left(\mathbb{A}_{\theta}-\lambda\right)^{-1} & \left(U+h\left(i \beta_{1},|\mathbf{x}|\right) \mathbf{x}^{t} \mathbf{u}_{1}\right) \\
= & \frac{1}{-\Delta-\lambda} U+\frac{1}{-\beta_{1}^{2}-\lambda} h\left(i \beta_{1},|\mathbf{x}|\right) \mathbf{x}^{t} \mathbf{u}_{1} \\
& -\frac{1}{Q(\lambda)+\cot \theta} \frac{h(k,|\mathbf{x}|)}{\lambda+\beta_{1}^{2}} \mathbf{x}^{t}\left(-\nabla\left(\frac{1}{-\Delta-\lambda} U\right)(0)+\frac{\gamma}{\beta_{1}^{2}+\lambda} \mathbf{u}_{1}\right) .
\end{aligned}
$$

As in the general case, it appears natural to consider the restriction of this 
resolvent to the infinite dimensional component $U \in W_{2}^{1}\left(\mathbb{R}^{3}\right) \subset \mathbb{H}$

$$
\begin{aligned}
& \text { 10.20) } \quad\left(\mathbb{A}_{\theta}-\lambda\right)^{-1} U=\frac{1}{-\Delta-\lambda} U \\
& -\frac{1}{\left(\lambda+\beta_{1}^{2}\right)(Q(\lambda)+\cot \theta)}\left(\int_{\mathbb{R}^{3}} \frac{(i k|\mathbf{y}|-1) e^{i k|\mathbf{y}|}}{4 \pi|\mathbf{y}|^{3}} \mathbf{y}^{t} U(\mathbf{y}) d^{3} \mathbf{y}\right) \frac{(i k|\mathbf{x}|-1) e^{i k|\mathbf{x}|}}{4 \pi|\mathbf{x}|^{3}} \mathbf{x} .
\end{aligned}
$$

It is hard not to notice the striking similarity of this form of the resolvent formula to Krein's original resolvent formula (2.11). Krein's $Q$-function should be replaced by the function appearing in the denominator of the last formula

$$
\begin{aligned}
Q_{1}(\lambda) & =\left(\lambda+\beta_{1}^{2}\right)\left(Q_{0}(\lambda)+\cot \theta\right) \\
& =\left(\lambda+\beta_{1}^{2}\right)\left(\frac{1}{12 \pi}\left\{i \sqrt{\lambda}+\frac{\beta_{1}^{2}}{i \sqrt{\lambda}-\beta_{1}}+\beta+\frac{\beta_{1}^{2}}{\beta+\beta_{1}}\right\}+\frac{\gamma}{-\beta_{1}^{2}-\lambda}+\cot \theta\right) .
\end{aligned}
$$

This function does not belong to the Nevanlinna class, since it grows like $\lambda^{3 / 2}$ as $\lambda \rightarrow \infty$. But it is a generalized Nevanlinna function, being the product of the Nevanlinna function $Q(\lambda)+\cot \theta$ with the polynomial $\lambda+\beta_{1}^{2}$ [13].

The operator $\mathbb{A}_{\theta}$ is a finite rank perturbation (in the resolvent sense) of the operator $\mathbb{A}_{0}=-\Delta \oplus-\beta_{1}^{2}$. Therefore, the spectrum of $\mathbb{A}_{\theta}$ contains the branch $[0, \infty)$ of the absolutely continuous spectrum (inherited from the Laplacian in $W_{2}^{1}\left(\mathbb{R}^{3}\right)$ ). In addition, the spectrum may contain several negative eigenvalues. The negative eigenvalues correspond to zeros of the $Q$-function on the negative axis. Let $\lambda_{0}<0$ be a solution of the equation

$$
Q\left(\lambda_{0}\right)+\cot \theta=0
$$

The function $Q(\lambda)$ is piecewise increasing with just a single singularity at $\lambda=-\beta_{1}^{2}$, and $Q(\lambda) \rightarrow_{\lambda \rightarrow-\infty}-\infty$. It follows that the $Q(\lambda)+\cot \theta$ has either one or two zeros on $\mathbb{R}_{-}$, depending on whether $Q(0)+\cot \theta=\frac{1}{12 \pi} \frac{\beta^{2}}{\beta+\beta_{1}}-\frac{\gamma}{\beta_{1}^{2}}+\cot \theta$ is less than or greater than zero, respectively. The corresponding eigenfunctions are given by

$$
\mathbb{V}_{\lambda_{0}}=h\left(k_{0},|\mathbf{x}|\right) \mathbf{x}^{t} \mathbf{a}=\frac{i k_{0}|\mathbf{x}|-1}{4 \pi|\mathbf{x}|^{3}} e^{i k_{0}|\mathbf{x}|} \mathbf{x}^{t} \mathbf{a}, \quad \lambda_{0}=k_{0}^{2}, i k_{0} \in \mathbb{R}_{-}
$$

where $\mathbf{a}=\left(a_{1}, a_{2}, a_{3}\right)^{t} \in \mathbb{C}^{3}$ is the vector parametrizing the three-dimensional space of eigenfunctions. Let us verify that this function is an eigenfunction for $\mathbb{A}_{\theta}$ provided $Q\left(\lambda_{0}\right)+\cot \theta=0$. First we show that $\mathbb{V}_{\lambda_{0}}$ possesses the representation 
$(10.10)$

$$
\begin{aligned}
\frac{i k_{0}|\mathbf{x}|-1}{4 \pi|\mathbf{x}|^{3}} & e^{i k_{0}|\mathbf{x}|} \mathbf{x}^{t} \mathbf{a} \\
= & -\frac{\beta_{1}|\mathbf{x}|+1}{4 \pi|\mathbf{x}|^{3}} e^{-\beta_{1}|\mathbf{x}|} \mathbf{x}^{t} \mathbf{a} \\
& +\left(\lambda+\beta_{1}^{2}\right) \frac{1}{\beta_{1}^{2}-\beta^{2}} \frac{-(\beta|\mathbf{x}|+1) e^{-\beta|\mathbf{x}|}+\left(\beta_{1}|\mathbf{x}|+1\right) e^{-\beta_{1}|\mathbf{x}|}}{4 \pi|\mathbf{x}|^{3}} \mathbf{x}^{t} \mathbf{a} \\
& +\left(\lambda+\beta_{1}^{2}\right)\left\{\frac{1}{\beta_{1}^{2}+k_{0}^{2}} \frac{\left(i k_{0}|\mathbf{x}|-1\right) e^{i k_{0}|\mathbf{x}|}+\left(\beta_{1}|\mathbf{x}|+1\right) e^{-\beta_{1}|\mathbf{x}|}}{4 \pi|\mathbf{x}|^{3}}\right. \\
& \left.-\frac{1}{\beta_{1}^{2}-\beta^{2}} \frac{-(\beta|\mathbf{x}|+1) e^{-\beta|\mathbf{x}|}+\left(\beta_{1}|\mathbf{x}|+1\right) e^{-\beta_{1}|\mathbf{x}|}}{4 \pi|\mathbf{x}|^{3}}\right\} \mathbf{x}^{t} \mathbf{a}
\end{aligned}
$$

It follows that the boundary values of $\mathbb{V}_{\lambda_{0}}$ are given by

$$
\left\{\begin{array}{l}
l \mathbf{v}_{1}=\mathbf{a} \\
\mathbf{v}=\left(\lambda_{0}+\beta_{1}^{2}\right) \mathbf{a} \\
-\nabla V_{r}(0)=\left(\lambda_{0}+\beta_{1}^{2}\right) \frac{1}{12 \pi}\left\{i k_{0}+\frac{\beta_{1}^{2}}{i k_{0}-\beta_{1}}+\beta+\frac{\beta_{1}^{2}}{\beta_{1}+\beta}\right\} \mathbf{a},
\end{array}\right.
$$

where $V_{r}$ denotes the last term in formula (10.23). It is easy to see that the boundary values satisfy the boundary conditions (10.12) due to (10.21). Since the action of the operator coincides with the action of the Laplacian, $\mathbb{V}_{\lambda_{0}}$ solves the equation $-\Delta \mathbb{V}_{\lambda_{0}}(\mathbf{x})-\lambda_{0} \mathbb{V}_{\lambda_{0}}(\mathbf{x})=0, \mathbf{x} \neq 0$, and clearly belongs to $\mathbb{H}$, we conclude that $\mathbb{V}_{\lambda_{0}}$ is an eigenfunction for $\mathbb{A}_{\theta}$. To recapitulate, the operator $\mathbb{A}_{\theta}$ has either one or two negative eigenvalues having multiplicity 3 , with eigenfunctions given by (10.22).

In a similar way, continuous spectrum eigenfunctions may be calculated. We start from the following Ansatz

$$
\mathbb{V}(\lambda, \mathbf{k} / k, \mathbf{x})=e^{i \mathbf{k}^{t} \mathbf{x}}+\frac{i k|\mathbf{x}|-1}{4 \pi|\mathbf{x}|^{3}} e^{i k|\mathbf{x}|} \mathbf{x}^{t} \mathbf{a}(\mathbf{k}), \quad \lambda>0,
$$

where $\mathbf{k} \in \mathbb{R}^{3},|\mathbf{k}|=k=\sqrt{\lambda}$, determines the direction of the incoming plane wave and the scattering amplitude $\mathbf{a}(\mathbf{k}) \in \mathbb{C}^{3}$ has to be calculated from the boundary conditions. Substituting the boundary values of $\mathbb{V}(\lambda)$

$$
\left\{\begin{array}{l}
\mathbf{v}_{1}=\mathbf{a} \\
\mathbf{v}=\left(\lambda+\beta_{1}^{2}\right) \mathbf{a} \\
-\nabla V_{r}(0)=-i \mathbf{k}+\left(\lambda+\beta_{1}^{2}\right) \frac{1}{12 \pi}\left\{i k+\frac{\beta_{1}^{2}}{i k-\beta_{1}}+\beta+\frac{\beta_{1}^{2}}{\beta_{1}+\beta}\right\} \mathbf{a}
\end{array}\right.
$$

into (10.12), we calculate the scattering amplitude a to be

$$
\mathbf{a}=\frac{i}{Q_{1}(\lambda)} \mathbf{k}
$$


which leads to the formula

$$
\mathbb{V}(\lambda, \mathbf{k} / k, \mathbf{x})=e^{i \mathbf{k}^{t} \mathbf{x}}+\frac{i}{Q_{1}(\lambda)} \frac{i k|\mathbf{x}|-1}{4 \pi|\mathbf{x}|^{3}} e^{i k|\mathbf{x}|} \mathbf{x}^{t} \mathbf{k}
$$

for the generalized eigenfunction corresponding to the absolutely continuous spectrum. Note that eigenfunctions constructed are not locally square integrable. The scattering matrix corresponding to this eigenfunction depends only on the energy and the angle between the directions of the incoming and outgoing waves, which shows yet again that the model is spherically symmetric. The model determines nontrivial scattering in the $p$-channel, since the scattering amplitude depends on the angle between the vectors $\mathbf{k}$ and $\mathbf{x}$. Rigorous proof of the eigenfunction expansion and spectral theorem for $\mathbb{A}_{\theta}$ can be carried out by integrating the jump of the resolvent at the real axis. The extended resolvent formula leads to a new family of eigenfunction expansions described, for example, in [24].

This model can be generalized to include point interactions in any other channel or a combination of such interactions in different channels. We plan to return to this question as well as to the spectral analysis of the operator $\mathbb{A}_{\theta}$ in the future. It might be interesting to study the relations between the model presented here and that suggested by Yu. Karpeshina [18].

Acknowledgements. The author thanks A. Luger for clarifying discussions and support during the preparation of the manuscript. He is also grateful to the referee for his careful reading of the manuscript and valuable remarks.

\section{REFERENCES}

[1] N. I. Akhiezer and I. M. Glazman, Theory of Linear Operators in Hilbert Space, Vol. I and II, Pitman, Boston, Mass.-London, 1981.

[2] S. Albeverio, F. Gesztesy, R. Høegh-Krohn and H. Holden, Solvable Models in Quantum Mechanics, 2nd edition. With an appendix by Pavel Exner. AMS Chelsea Publishing, Providence, RI, 2005.

[3] S. Albeverio and P. Kurasov, Rank one perturbations, approximations, and self-adjoint extensions, J. Funct. Anal. 148 (1997), 152-169.

[4] S. Albeverio and P. Kurasov, Rank one perturbations of not semibounded operators, Integral Equations Operator Theory 27 (1997), 379-400.

[5] S. Albeverio and P. Kurasov, Singular Perturbations of Differential Operators. Solvable Schrödinger Type Operators, Cambridge University Press, Cambridge, 2000.

[6] A. Alonso and B. Simon, The Birman-Krein-Vishik theory of self-adjoint extensions of semibounded operators, J. Operator Theory 4 (1980), 251-270.

[7] F. A. Beresin and L. D. Faddeev, A remark on Schrödinger equation with a singular potential, Sov. Math. Doklady 137 (1961), 1011-1014.

[8] Yu. M. Berezanskiǔ, Expansions in Eigenfunctions of Selfadjoint Operators, Amer. Math. Soc., Providence, RI, 1968. 
[9] M. S. Birman, On the self-adjoint extensions of positive definite operators, Mat. Sb. (N.S.) 38 (80) (1956), 431-456.

[10] J. W. Calkin, Abstract symmetric boundary conditions, Trans. Amer. Math. Soc. 45 (1939), 369-442.

[11] Yu. Demkov and V. Ostrovsky, Zero-range Potentials and their Applications in Atomic Physics, Plenum, New York, 1988.

[12] A. Dijksma, P. Kurasov and Yu. Shondin, High order singular rank one perturbations of a positive operator, Integral Equations Operator Theory 53 (2005), 209-245.

[13] A. Dijksma, H. Langer, A. Luger and Yu. Shondin, A factorization result for generalized Nevanlinna functions of the class $\mathcal{N}_{\kappa}$, Integral Equations Operator Theory 36 (2000), 121-125.

[14] A. Dijksma, H. Langer, Yu. Shondin and C. Zeinstra, Self-adjoint operators with inner singularities and Pontryagin spaces, in Operator Theory and Related Topics, vol. II, Birkhäuser, Basel, 2000, pp. 105-175.

[15] A. Dijksma and Yu. Shondin, Singular point-like perturbations of the Laguerre operator in a Pontryagin space, in Operator Methods in Ordinary and Partial Differential Equations, Birkhäuser, Basel, 2002, pp. 141-18.

[16] E. Fermi, Sul moto dei neutroni nelle sostanze idrogenate, Ricerca Scientifica, 7 (1936), 13-52, English translation in E. Fermi, Collected Papers, Vol. I, Italy 1921-1938, University of Chicago Press, Chicago, 1962, pp. 980-1016.

[17] F. Gesztesy and B. Simon, Rank one perturbations at infinite coupling, J. Funct. Anal. 128 (1995), 245-252.

[18] Yu. E. Karpeshina, Zero-range model of p-scattering by a potential well, Forschungsinstitut für Mathematik, ETH, Zürich, 1992, preprint.

[19] A. Kiselev and B. Simon, Rank one perturbations with infinitesimal coupling, J Funct. Anal. 130 (1995), 345-356.

[20] M. Krein, On Hermitian operators whose deficiency indices are 1, C. R. (Doklady) Acad. Sci. URSS (N.S.) 43 (1944), 323-326.

[21] M. Krein, The theory of self-adjoint extensions of semibounded Hermitian transformations and its applications, I, Rec. Math. (Mat. Sb.) 20 (62) (1947), 431-495.

[22] P. Kurasov, $\mathcal{H}_{-n}$-perturbations of self-adjoint operators and Krein's resolvent formula, Integral Equations Operator Theory 45 (2003), 437-460.

[23] P. Kurasov, Singular and supersingular perturbations: Hilbert space methods, in Spectral Theory of Schrödinger Operators, Amer. Math. Soc., Providence, RI, pp. 185-216.

[24] P. Kurasov and A. Luger, Singular differential operator: Titchmarsh-Weyl coefficients and operator models, Report N8, Dept. of Math., Lund Univ., 2007.

[25] P. Kurasov and K. Watanabe, On rank one $\mathcal{H}_{-3}$-perturbations of positive self-adjoint operators, in Stochastic Processes, Physics and Geometry: New Interplays, II, Amer. Math. Soc., Providence, 2000, pp. 413-422.

[26] P. Kurasov and K. Watanabe, On $\mathcal{H}_{-4}$-perturbations of self-adjoint operators, in Partial Differential Equations and Spectral Theory, Birkhäuser, Basel, 2001, pp. 179-196.

[27] J. von Neumann, Allgemeine Eigenwettheorie Hermitescher Funktionaloperatoren, Math. Ann. $102(1929-30), 49-131$.

[28] B. Pavlov, The theory of extensions and explicitly solvable models, Uspekhi Mat. Nauk 42 (1987), 99-131.

[29] B. Pavlov, Boundary conditions on thin manifolds and the semiboundedness of the three-body Schrödinger operator with point potential, Mat. Sb. (N.S.) 136 (178) (1988), 163-177.

[30] M. Reed and B. Simon, Methods of Modern Mathematical Physics II, Academic Press, New York, 1972. 
[31] B. Simon, Spectral analysis of rank one perturbations and applications, CRM Proceedings and Lecture Notes 8 (1995), 109-149.

[32] M. Vishik, On general boundary condition for elliptic differential equation, Trudy Moskov. Mat. Obshch. 1 (1952), 187-246.

\author{
Pavel Kurasov \\ DEPARTMENT OF MATHEMATICS \\ LTH, LUND UNIVERSITY \\ BoX 118, 22100 LUND, SWEDEN \\ email: kurasov@maths.Ith.se \\ and \\ DEPARTMENT OF MATHEMATICS \\ STOCKHOLM UNIVERSITY \\ 10691 STOCKHOLM, SWEDEN \\ and \\ DEPARTMENT OF MATHEMATICS \\ St. PETERSBURG UNIVERSITY \\ 198904 ST. PETERHOF, RUSSIA
}

(Received December 23, 2007) 